\title{
Determination of precise crystallographic directions for mask alignment in wet bulk micromachining for MEMS
}

\author{
Sajal Sagar Singh ${ }^{1 *}$, Prem Pal ${ }^{2}$, Ashok Kumar Pandey $^{1}$, Yan Xing ${ }^{3}$ and Kazuo Sato ${ }^{4}$
}

\begin{abstract}
In wet bulk micromachining, the etching characteristics are orientation dependent. As a result, prolonged etching of mask openings of any geometric shape on both $\mathrm{Si}\{100\}$ and $\mathrm{Si}\{110\}$ wafers results in a structure defined by the slowest etching planes. In order to fabricate microstructures with high dimensional accuracy, it is vital to align the mask edges along the crystal directions comprising of these slowest etching planes. Thus, precise alignment of mask edges is important in micro/nano fabrication. As a result, the determination of accurate crystal directions is of utmost importance and is in fact the first step to ensure dimensionally accurate microstructures for improved performance. In this review article, we have presented a comprehensive analysis of different techniques to precisely determine the crystallographic directions. We have covered various techniques proposed in the span of more than two decades to determine the crystallographic directions on both $\mathrm{Si}\{100\}$ and Si\{110\} wafers. Apart from a detailed discussion of each technique along with their design and implementation, we have provided a critical analysis of the associated constraints, benefits and shortcomings. We have also summed up the critical aspects of each technique and presented in a tabular format for easy reference for readers. This review article comprises of an exhaustive discussion and is a handy reference for researchers who are new in the field of wet anisotropic etching or who want to get abreast with the techniques of determination of crystal directions.
\end{abstract}

\section{Background}

Micromachining is an integral part of micro/nanofabrication for MEMS/NEMS industry [1-20]. There are two kinds of micromachining methods namely surface micromachining and bulk micromachining [17, 19]. As the name indicates, surface micromachining technique makes use of the surface of the substrate (e.g., wafer) and the micro/nanostructures are fabricated using deposited thin films on the surface. The deposited thin films are used as structural and sacrificial layers [9-18]. Bulk micromachining on the other hand selectively etches the bulk to fabricate 3-D features, suspended beams, membranes, etc. $[1-16,19,20]$. Bulk micromachining is further divided into two categories: dry and wet etching. Dry etching is mainly preformed using gas phase plasma

\footnotetext{
*Correspondence: me11b028@iith.ac.in

${ }^{1}$ Department of Mechanical and Aerospace Engineering, Indian Institute of Technology Hyderabad, Kandi Sangareddy, India

Full list of author information is available at the end of the article
}

[21-26], but focussed ion beam and laser machining are also used in some special cases $[27,28]$. If the etching is performed using wet chemicals, it is termed as wet etching. Based on the etch rates in different directions, wet etching can be further sub-divided into isotropic and anisotropic etching. In isotropic etching, the etch rate is same in all directions and does not depend on the crystallographic directions, however in the case of anisotropic etching the etch rate is a function of the crystallographic orientation [9-14]. Common silicon wet isotropic etchants are mixture of $\mathrm{HF}, \mathrm{HNO}_{3}$ and $\mathrm{CH}_{3} \mathrm{COOH}[29,30]$, while potassium hydroxide $(\mathrm{KOH})[2,7,31-43]$ and tetramethylammonium hydroxide (TMAH) $[1,5,35$, 44-64] etchants are most extensively used for wet anisotropic etching. There are some other alkaline solutions which have been investigated for silicon wet anisotropic etching such as ethylenediamine pyrocatechol water (EDP or EPW) [4, 35, 41, 65-67], hydrazine [31, 68, 69], ammonium hydroxide [70], and cesium hydroxide 
$(\mathrm{CsOH})$ [71]. In wet anisotropic etching, $\{111\}$ planes are the slowest etch rate planes in all types of anisotropic etchants. Therefore a stable etched profile (or prolonged etched profile) in silicon wafer is formed by $\{111\}$ planes, for instance, an arbitrary shaped mask opening on $\operatorname{Si}\{100\}$ wafer leads to a square/rectangular V-groove comprising of $\{111\}$ planes at the four mutually perpendicular $\langle 110\rangle$ directions as shown in Fig. 1 [72], while on $\mathrm{Si}\{110\}$ wafer it gives a hexagonal groove type of structure comprising of $\{111\}$ planes at the $\langle 110\rangle$ and $\langle 112\rangle$ directions as presented in Fig. 2 [73].

\section{Role of crystallographic alignment in wet bulk micromachining}

In surface micromachining, there are generally no issues of proper alignment of the mask edges/sides along crystallographic directions as the wafer/substrate is used only as a support for the fabrication of micro/nanostructures.

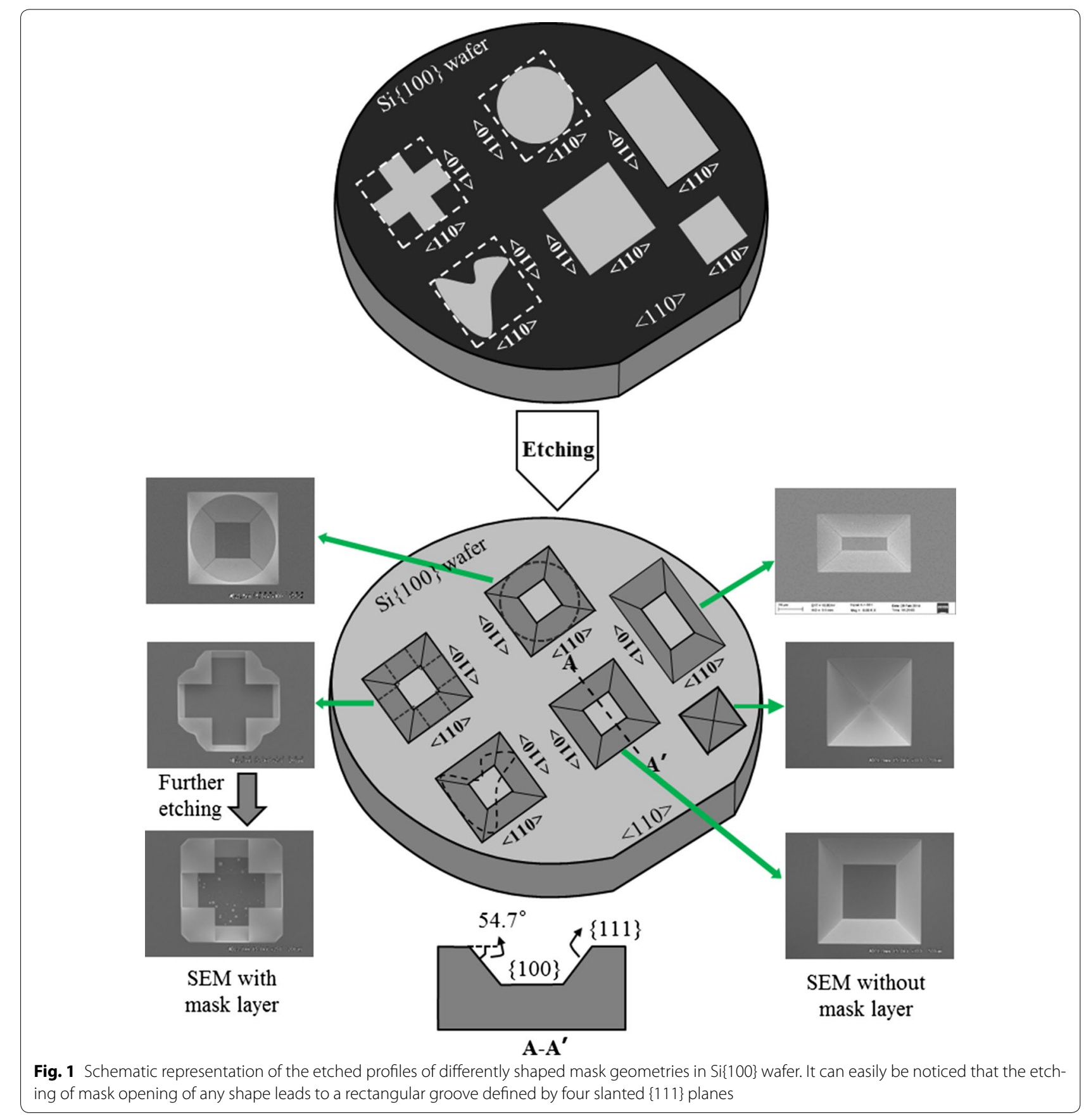




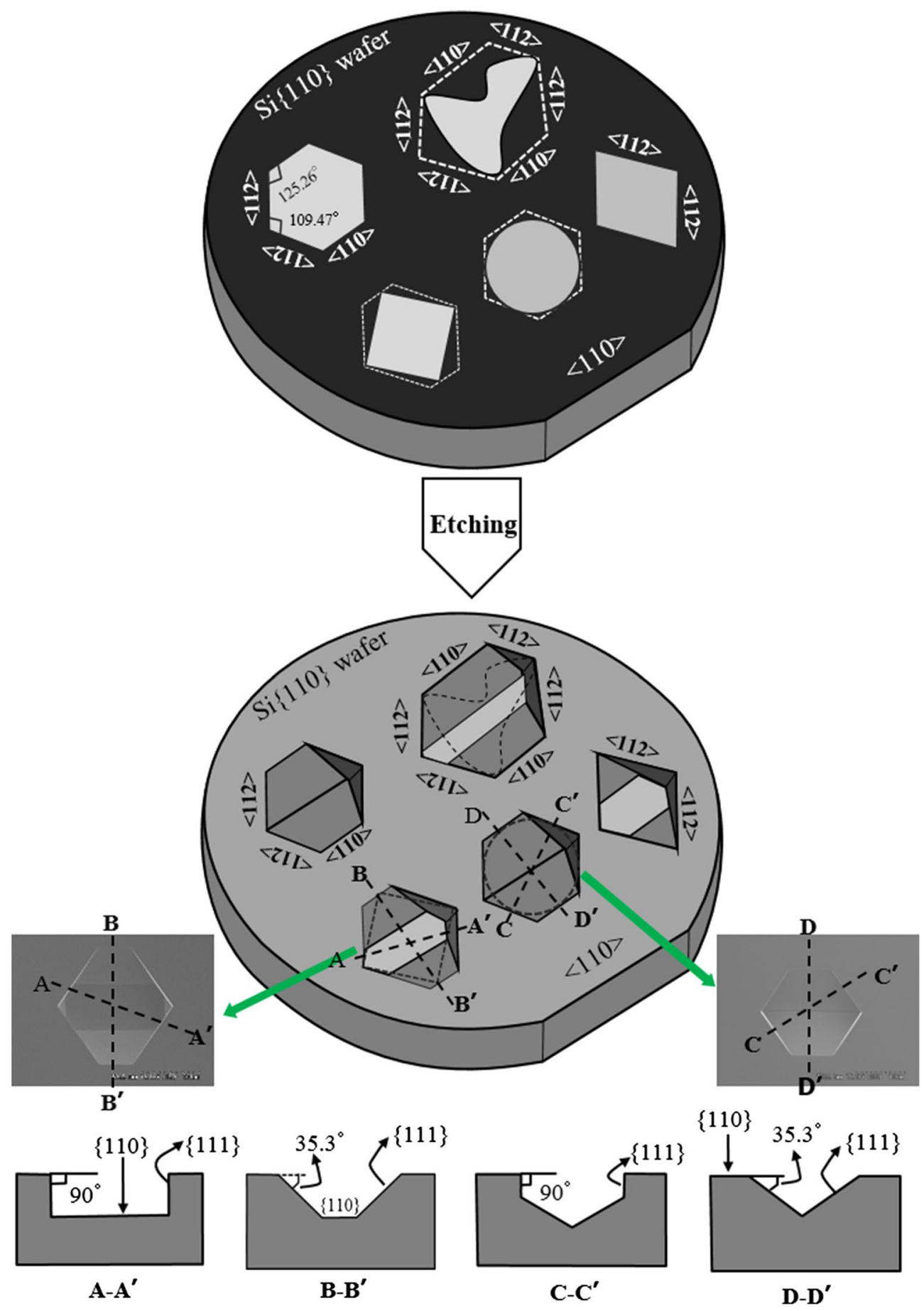

Fig. 2 Schematic representation of the etched profiles of differently shaped mask geometries in Si\{110\} wafer. It can be seen that the etching of mask opening of any shape leads to a hexagonal groove defined by two slanted and four vertical $\{111\}$ planes

Similarly, dry etching, which although uses the bulk material for fabrication is almost orientation independent, therefore precise alignment of the mask patterns along the crystallographic directions is also not of much importance. In the case of isotropic etching, the etched profile is orientation independent. Therefore the precise alignment of mask geometries with crystallographic directions does not attract much importance in this type 
of etching as well. However, on the contrary to these types of etching, anisotropic wet etching is highly orientation dependent. As a result it is of utmost significance to ensure that the mask edges are aligned precisely along the required crystallographic directions in order to fabricate dimensionally accurate microstructures. This makes the precise determination of crystallographic orientation a vital step to avoid over sizing of the structure and to obtain smooth sidewalls. The oversizing of the structures depends on the degree of the misalignment as well as the length of the structures. The importance of precise alignment in wet anisotropic etching based silicon micromachining is described by taking three examples of the most widely used structures in MEMS namely microchannel, cantilever beam and diaphragm.

\section{Microchannels}

Microchannels are extensively employed in bio-MEMS and micro-fluidics applications. The lengths of these micro-channels vary from a few microns to several millimetres [19, 74-77]. In order to fabricate a dimensionally accurate vertical micro-channel in $\{110\}$ silicon wafers, the mask edges must be aligned precisely along the $\langle 112\rangle$ directions as the stable and vertical $\{111\}$ planes appear at these directions. When the mask edges are aligned precisely along the crystal direction, the channels are distinctly defined as per their dimensions. However, even a small misalignment would lead to undercutting as shown in Fig. 3. If the channels are patterned closely to each other, the undercutting due to misalignment can also lead to merging of the channels.

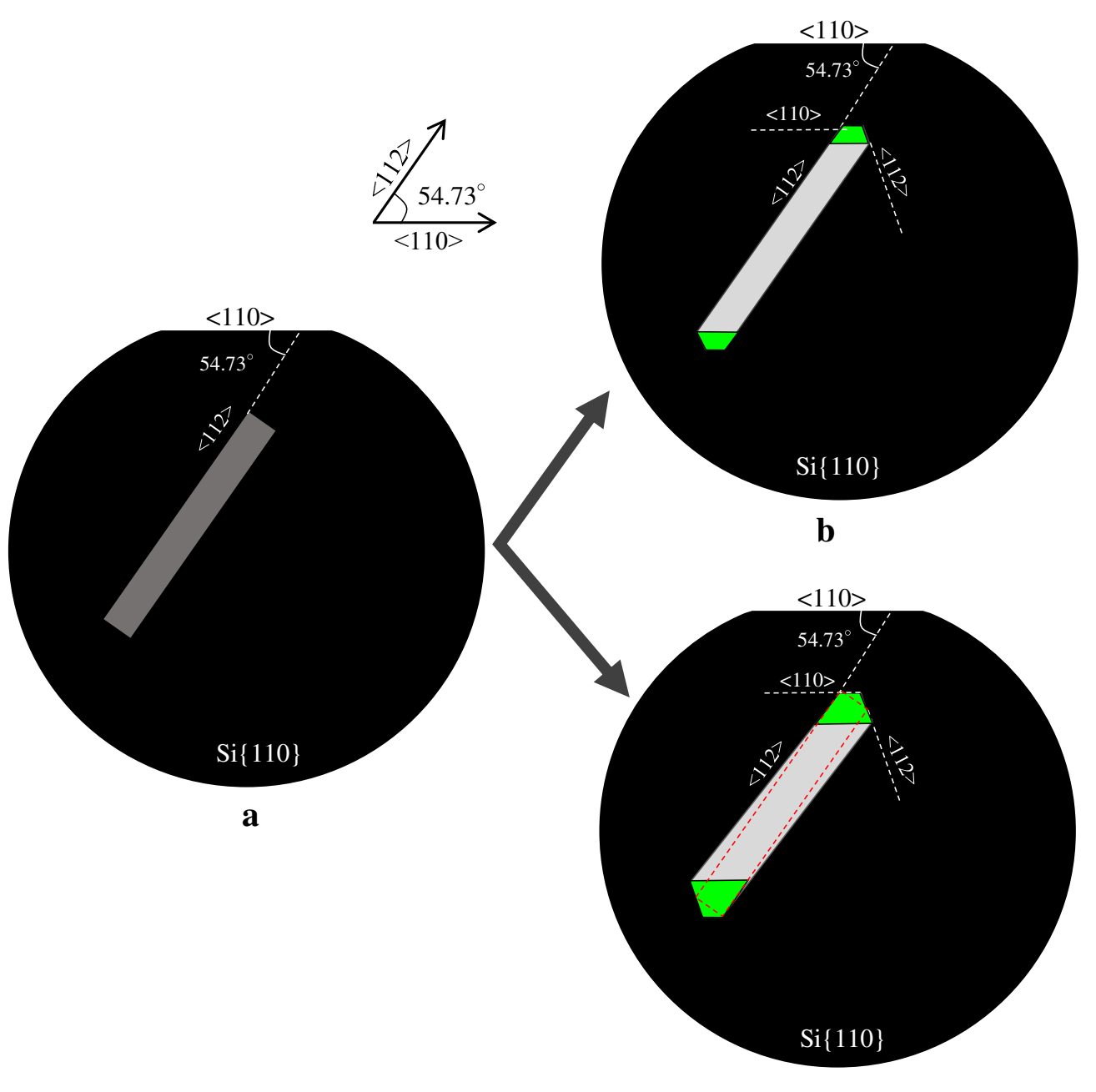

c

Fig. 3 Effect of the misalignment of mask edges on the etched profile on Si\{1 10\} wafers: a patterning of a channel shape mask design using the wafer flat as the reference $\langle 110\rangle$ direction, $\mathbf{b}$ precise alignment of long edges along $\langle 112\rangle$ directions, $\mathbf{c}$ misalignment of long edges leading to oversizing of the channel 
Similarly, long cavities formed on $\mathrm{Si}\{100\}$ wafer gets oversized due to misalignment by even a small angle. It happens due to the undercutting at misaligned mask edges as shown schematically in Fig. 4. Although there would be some undercutting even in the case of precisely aligned mask owing to the finite etch rate of $\{111\}$ plane, however this undercutting is extremely less to cause any significant oversizing of the channels or cavities. The amount of undercutting $(U)$ (measured perpendicular to the mask edge) due to misalignment by an angle $\delta$ for length $l$, as presented in Fig. 4, can be calculated using a simple trigonometric relation as follows:

$$
U=l \sin (\delta) \cos (\delta)
$$

Thus, a microchannel of length $10 \mathrm{~mm}$ will result in an undercut of $\sim 18 \mu \mathrm{m}$ even for a misalignment of $0.1^{\circ}$.

\section{Cantilever beams}

The diodes and transistors are considered as basic building blocks of integrated circuits (ICs). In MEMS, the shape and size of the structures are application dependent. Therefore there are no elementary building blocks which can support all designs. However, cantilevers and diaphragms are extensively used for sensing and actuation purposes in wide variety of applications including but not limited to bio-sensors, switches, temperature sensor and many others [78-99]. Thus they can be called as the fundamental structures of MEMS devices. Arrays of cantilever beams are used in various sensors and

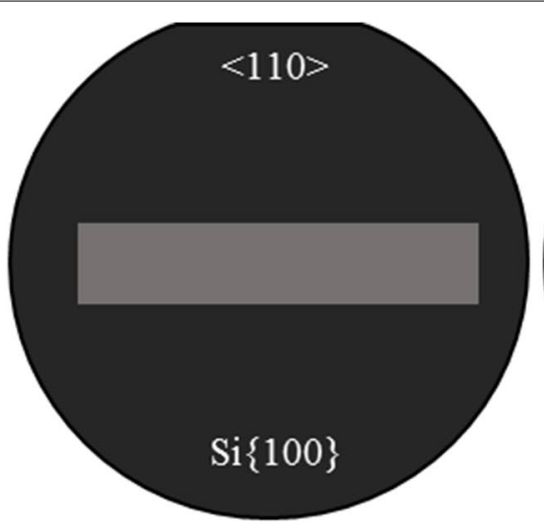

a

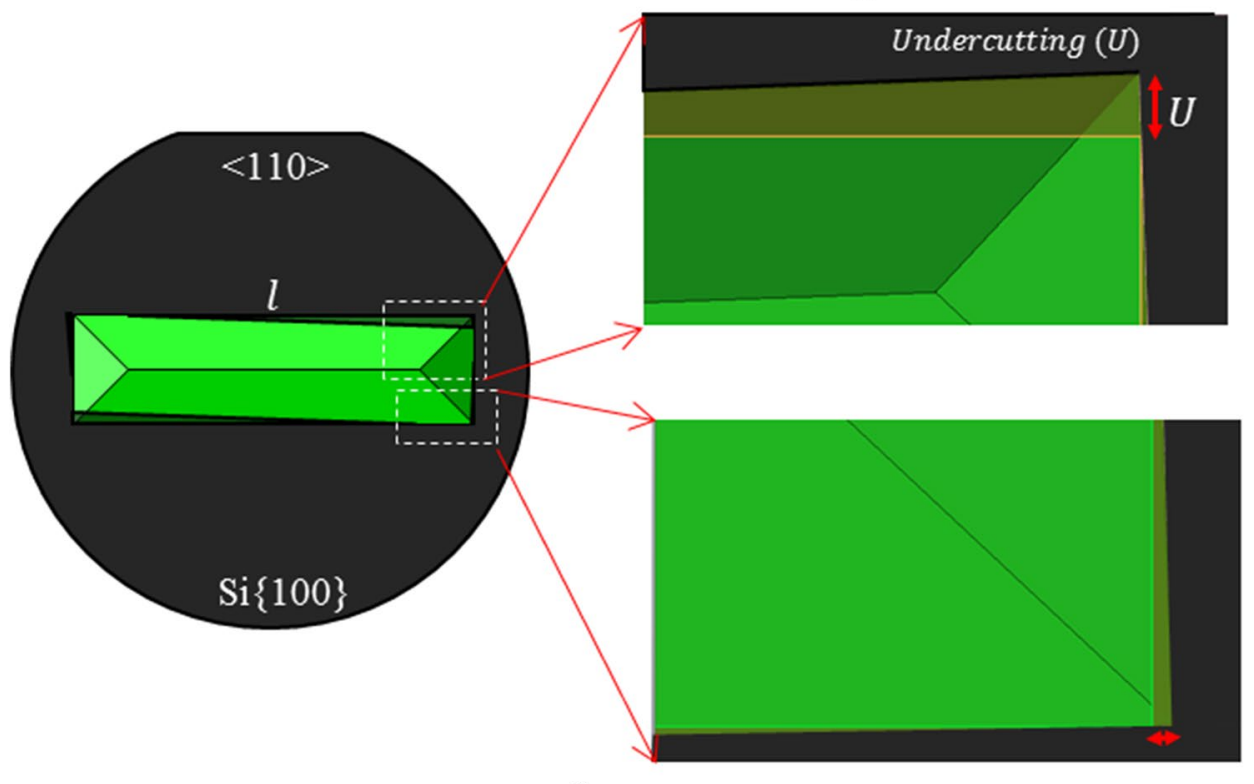

c

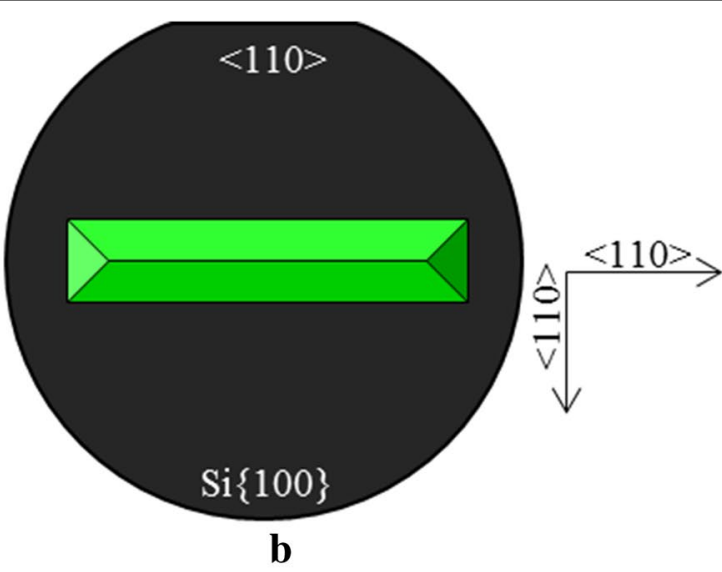

Undercutting $(U)$

Fig. 4 Effect of the misalignment of mask edges on the etched profile on Si\{100\} wafer: a channel (or rectangular opening) patterned using the wafer flat as the reference $\langle 110\rangle$ directions. Etched profile of the channel when the mask edges are $\mathbf{b}$ accurately and $\mathbf{c}$ inaccurately oriented along $\langle 110\rangle$ directions. In the case of accurate alignment, the etched channel is dimensionally accurate without any undercutting, while undercutting occurs if the mask edges are misaligned 
actuators for developing high performance devices such as artificial nose [84]. These arrays are fabricated either by front side etching or backside etching. In order to fabricate using front side etching, the phenomenon of convex corer undercutting is used for their release from substrate $[8,100]$. Etching stops when it encounters the $\{111\}$ planes at the anchoring point of the beam.

Thus precise alignment of the mask edges along crystallographic directions is necessary to obtain dimensionally accurate cantilever beams as shown in Fig. 5a. Owing to large number of beams, even a slight misalignment would result in larger underetching at the anchor points leading to additional overhang as shown in Fig. 5b. The additional overhang results in coupling of the beams and affects their dynamic characteristics. In order to fabricate high performance devices, it is necessary to avoid such coupling and align the mask patterns precisely along the $\langle 110\rangle$ direction on $\operatorname{Si}\{100\}$.

\section{Diaphragm}

Another important MEMS structure used in sensing devices is diaphragm. To fabricate the diaphragm, the etching is done throughout the wafer thickness $(h)$ from the backside as shown schematically in Fig. 6 . The length of the mask pattern (b) for fabricating a square diaphragm of sides $l$ can be calculated using following formula:

$$
b=\frac{(l+2 h \cot (54.7))}{(\sin (\delta)+\cos (\delta))}
$$

where, $\delta$ is the misaligned angle. It is evident from this formula that the size of the mask edge $(b)$ for a particular diaphragm length $(l)$ depends on the misalignment angle $\delta$. In order to control the dimensions of the diaphragm one need to ensure that $\delta$ should be negligible. In quantitative terms, to fabricate a square diaphragm of side $l=50 \mu \mathrm{m}$ through a $500 \mu \mathrm{m}$ thick $\operatorname{Si}\{100\}$ wafer, the sides of the mask opening should be around $758.04 \mu \mathrm{m}$. However, misalignment by half degree would make the diaphragm around $13 \%$ more of its expected dimensions which can alter the performance of a sensor.

Owing to the importance of fabricating dimensionally accurate structures with smooth sidewalls, study of crystallographic alignment techniques is extremely vital in the field of wet anisotropic etching based bulk micromachining. Another benefit of accurately aligned mask is that it reduces the etching time necessary for obtaining smooth sidewalls. While there have been several review articles published in various areas of microfabrication
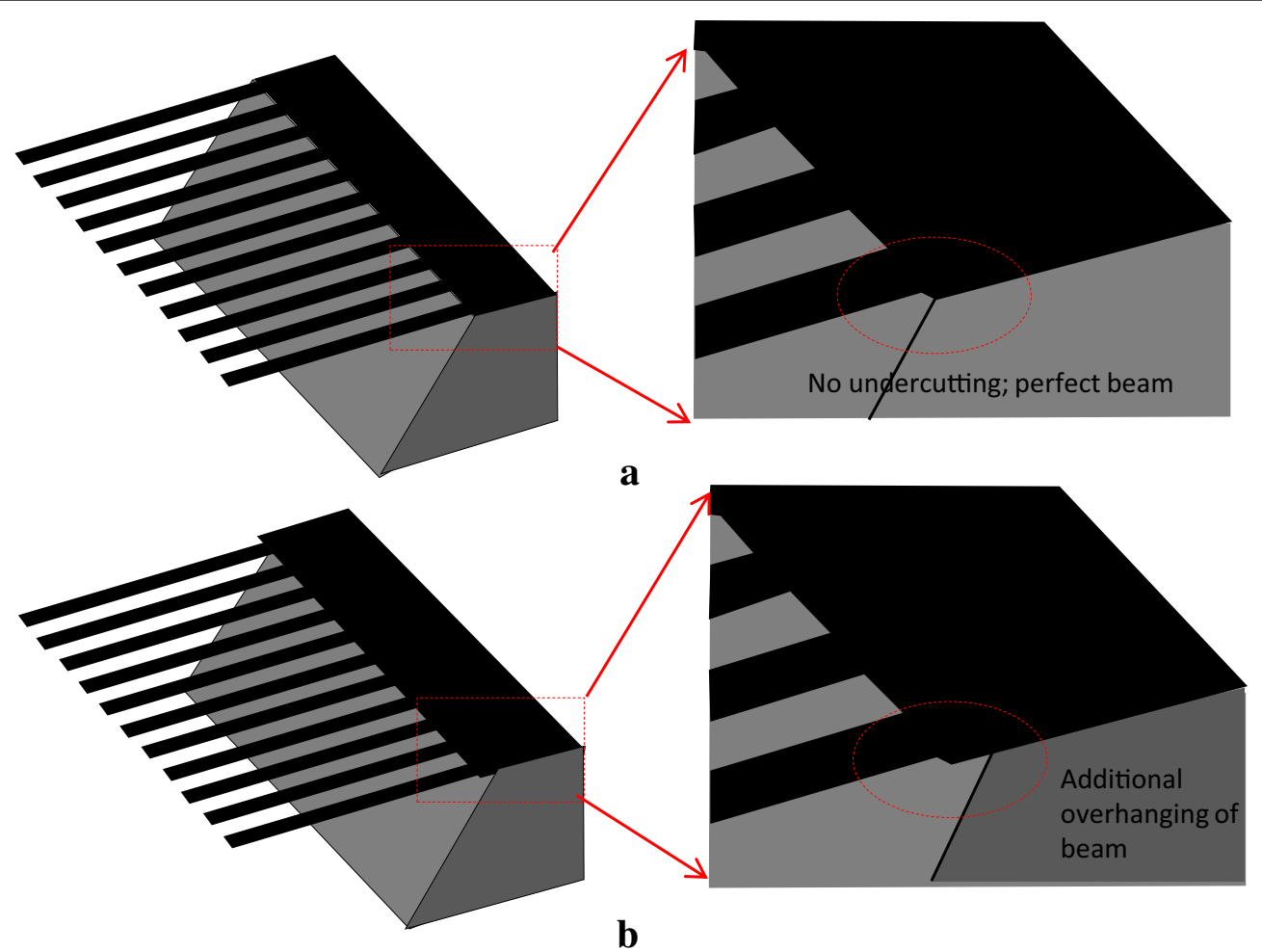

Fig. 5 Schematic diagram of an array of cantilever beams fabricated when the mask edges are aligned along $\langle 110\rangle$ direction $\mathbf{a}$ accurately and $\mathbf{b}$ inaccurately. The zoomed view shows that there is no underetching and oversizing (or additional overhang) when the mask is accurately aligned with $\langle 110\rangle$ direction, while additional overhang occurs due to the misalignment of mask patterns. The additional overhanging leads to coupling between adjacent beams 


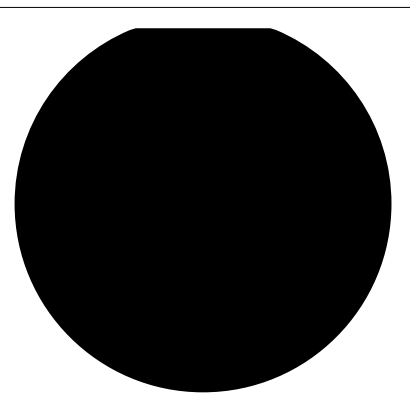

$\mathbf{a}$

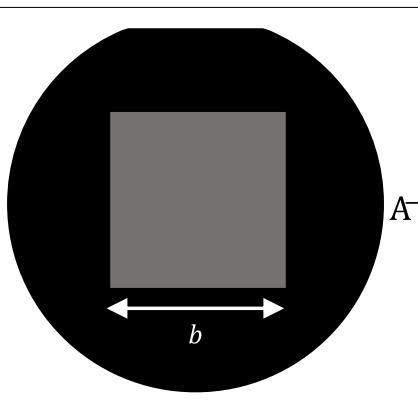

b

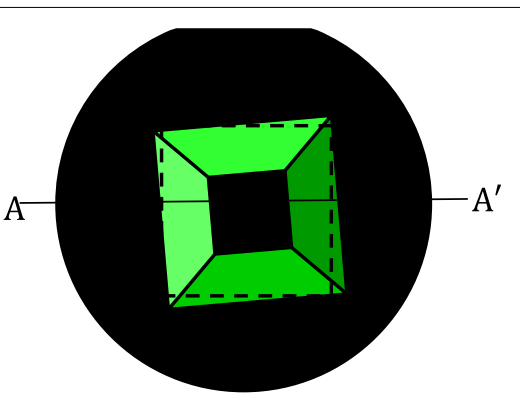

c

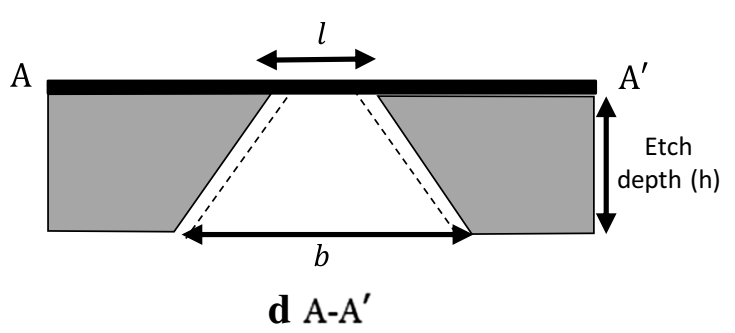

Fig. 6 Schematic view showing the fabrication of diaphragm on $\mathrm{Si}\{100\}$ wafer: a deposition of thin film of diaphragm material (e.g. $\left.\mathrm{SiO}_{2}\right)$, b patterning of backside, $\mathbf{c}$ wet anisotropic etching, $\mathbf{d}$ cross sectional view of the suspended diaphragm. The dotted lines show the geometry when the mask edges are aligned perfectly along the crystallographic direction, while solid line indicates the over etched geometry due to misalignment

including wet etching $[8,100]$, dry etching [21], microvalves [92], micropumps [95, 97], microfluidics [101], etc., there is no review article till date which extensively discusses various techniques to identify different crystallographic directions on silicon wafers along with their associated pros and cons. Given the importance of precise alignment as discussed above, it is very important to understand the role of alignment as well as the methodologies to ensure precise mask alignment. This paper is a comprehensive review of all the proposed pre-etched patterns published from the last two decades till date. It deals with the pros and cons of various pre-etched structures as well as which design patterns are easy to fabricate and are less involved in analysing them when it comes to determining the accurate crystallographic directions. We start with a brief discussion on the methods used for aligning the mask edges to the crystallographic directions. Subsequently, the role of different pre-etched patterns in identifying the crystal directions along with the merits of each technique is discussed.

\section{Alignment techniques}

Basic method to align the mask patterns along crystallographic directions is by using the wafer flat as the reference crystallographic direction. Generally the wafer manufacturing industries uses an optical beam to focus at etched surface and the reflected ray is used to determine the crystallographic direction (wafer flat) of the whole ingot prior to cutting it to wafers [102]. These wafer flats usually have a crystallographic misalignment ranging from $1^{\circ}$ to $5^{\circ}$ depending on the quality of the wafer [103]. An inaccurate wafer flat would lead to inaccuracy in aligning the mask edges along crystallographic directions leading to undesired increase in dimensions as discussed in previous section (refer Figs. 3, 4). For example, in order to fabricate a vertical walled channel on $\mathrm{Si}\{110\}$ wafer, we need to align the channel mask precisely along $\langle 112\rangle$ direction. However, if the flat is misaligned, the mask edges will not be aligned along the $\langle 112\rangle$ directions. This will lead to undercutting resulting in an inaccurately dimensioned (oversized) channel. This inaccuracy is undesirable for the high performance devices used today as they require extremely high precision control of dimensions. Thus the wafer flat used for aligning mask is no longer sufficient to achieve the high precision. Another method is to use X-ray diffraction, which can exactly determine the crystallographic direction, but it is highly impracticable to mount the diffraction equipment onto the mask aligner. As a result there have been other attempts to determine the crystallographic directions with high accuracy on $\operatorname{Si}\{100\}$ and $\operatorname{Si}\{110\}$ wafers which are most widely used in semiconductor laboratories and industries.

In order to determine the crystallographic directions (e.g., $\langle 110\rangle)$ on silicon wafer with high precision either cleaved-edge method or wet anisotropically pre-etched patterns are employed. These methods are described in following subsections. 


\section{Cleaved-edge alignment}

Single-crystal materials have a tendency to cleave along crystallographic planes and are caused by the alignment of weaker bonds between atoms in the crystal lattice. In single crystalline silicon, a perfect cleavage takes places at the direction comprising $\{111\}$ and $\{110\}$ planes as the bond density for these planes is lower than other planes such as $\{100\}$. Therefore, the slip lines and other defects at the edges of silicon wafers are usually responsible for wafer breakage. In this method, the silicon wafer is cleaved to reveal $\{111\}$ planes for alignment, for instance, cleaved edges on $\{110\}$ wafer are aligned along $\langle 110\rangle$ and $\langle 112\rangle$ directions as shown in Fig. 7 for the wafer whose flat is oriented along $\langle 110\rangle$ direction. In order to fabricate the microstructures, for example long and deep channels on $\{110\}$ wafer, the edges of the microchannel patterns are aligned with cleaved-edges oriented along $\langle 112\rangle$ directions [76]. Although this method is very simple to identify the crystallographic directions, wafer is separated into pieces and the cleaved surface may not be formed by a single $\{111\}$ planes instead a rugged surface is obtained as can be seen in Fig. 8a, c [76]. This leads to very poor results as can be seen in the experimental images presented in Fig. 8b [76]. Therefore, this method of alignment is undesirable owing to the uncertainty in obtaining the cleaving along the precise crystal direction. Besides, reproducibility of this method is questionable.

\section{Pre-etched patterns}

The cleaved-edge method relies on the accuracy of the wafer flat as well as the handler's accuracy to cleave (or dice) the wafer along the scribed line. Additionally, it also depends on the accuracy of the scribed directions.

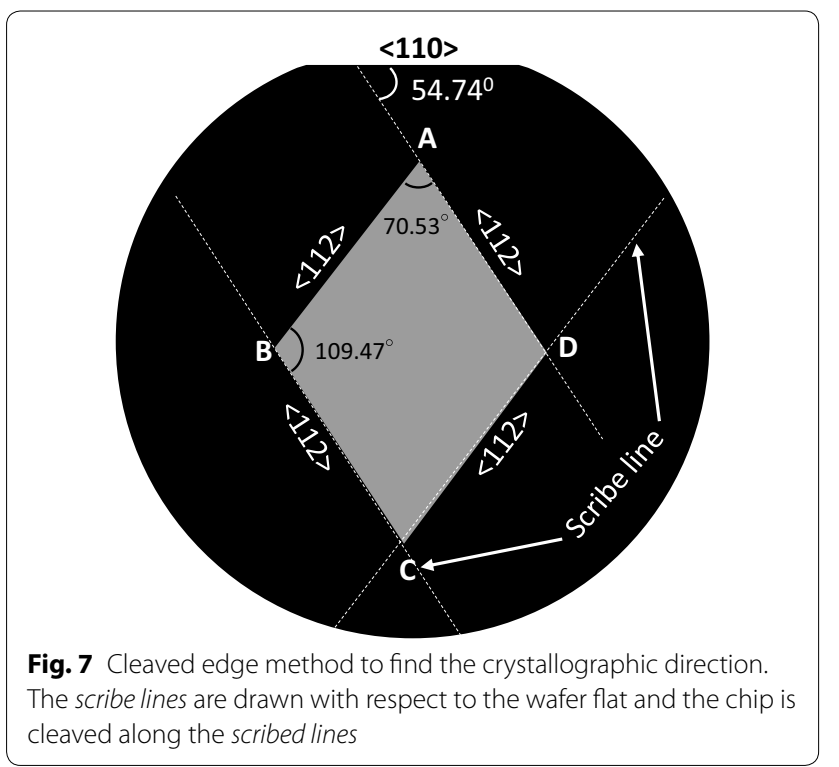

Therefore, this is not an optimal method to ensure precise determination of crystallographic direction. As a result, more accurate techniques are needed for the accurate determination of crystal directions.

Researchers in the past have used anisotropic etching to fabricate patterns to determine the crystallographic direction (e.g., $\langle 110\rangle,\langle 100\rangle)$ with high precision. These patterns are etched on the periphery of the wafer and therefore the usable wafer space is not affected significantly. These preetched patterns act as aids for the subsequent alignment of mask edges. This method of alignment has been accurate in determining the crystallographic directions precisely with an accuracy as high as $0.01^{\circ}$. This has helped the researchers in fabricating dimensionally accurate micro-structures for different applications. Many designs have previously been proposed which talks about the alignment techniques and its accuracy. Several pre-etched patterns have been proposed till date using various geometries [76, 102-110]. Some of them aim at determining the $\langle 110\rangle$ direction on $\mathrm{Si}\{100\}$ and $S\{110\}$ wafers $[76,102,104-107]$. At the same time, some have been aimed at determining the $\langle 100\rangle$ direction on both types of wafer $[103,108,109]$. In the subsequent subsections, we discuss various techniques, design geometry and their accuracies in determining the crystallographic directions which have been published in the period of more than two decades. Apart from high accuracy, a good pre-etched pattern should have minimum measurement requirement, should be visually identifiable with simple equipment and should not use much of the usable wafer space. At the same time it should not only determine the crystallographic directions but also aid in alignment of the subsequent mask. We categorise the techniques based on their suitability for wafers of different orientations. Some techniques exploit the shape of etched profiles and thus can be exclusively used for one particular wafer orientation. However, some techniques have been proposed which can be used for different types of wafers. In the subsequent subsections, we discuss the details of the patterns starting with the patterns for determining directions on $\operatorname{Si}\{100\}$ followed by $\operatorname{Si}\{110\}$ wafers.

\section{Identification of crystallographic directions on Si\{100\} wafer}

In the fabrication of microstructures on $\mathrm{Si}\{100\}$ wafer using wet anisotropic etching, identification of $\langle 110\rangle$ direction is of interest due to the appearance of stable $\{111\}$ planes at these directions. Nevertheless, $\langle 100\rangle$ directions are also important due to the appearance of stable $\{110\}$ planes when etching is done in surfactant added etchants. These $\{110\}$ planes are used as $45^{\circ}$ micromirrors in optical MEMS applications. We start with the techniques to determine the precise $\langle 110\rangle$ directions followed by a discussion on the techniques to determine the $\langle 100\rangle$ directions. 


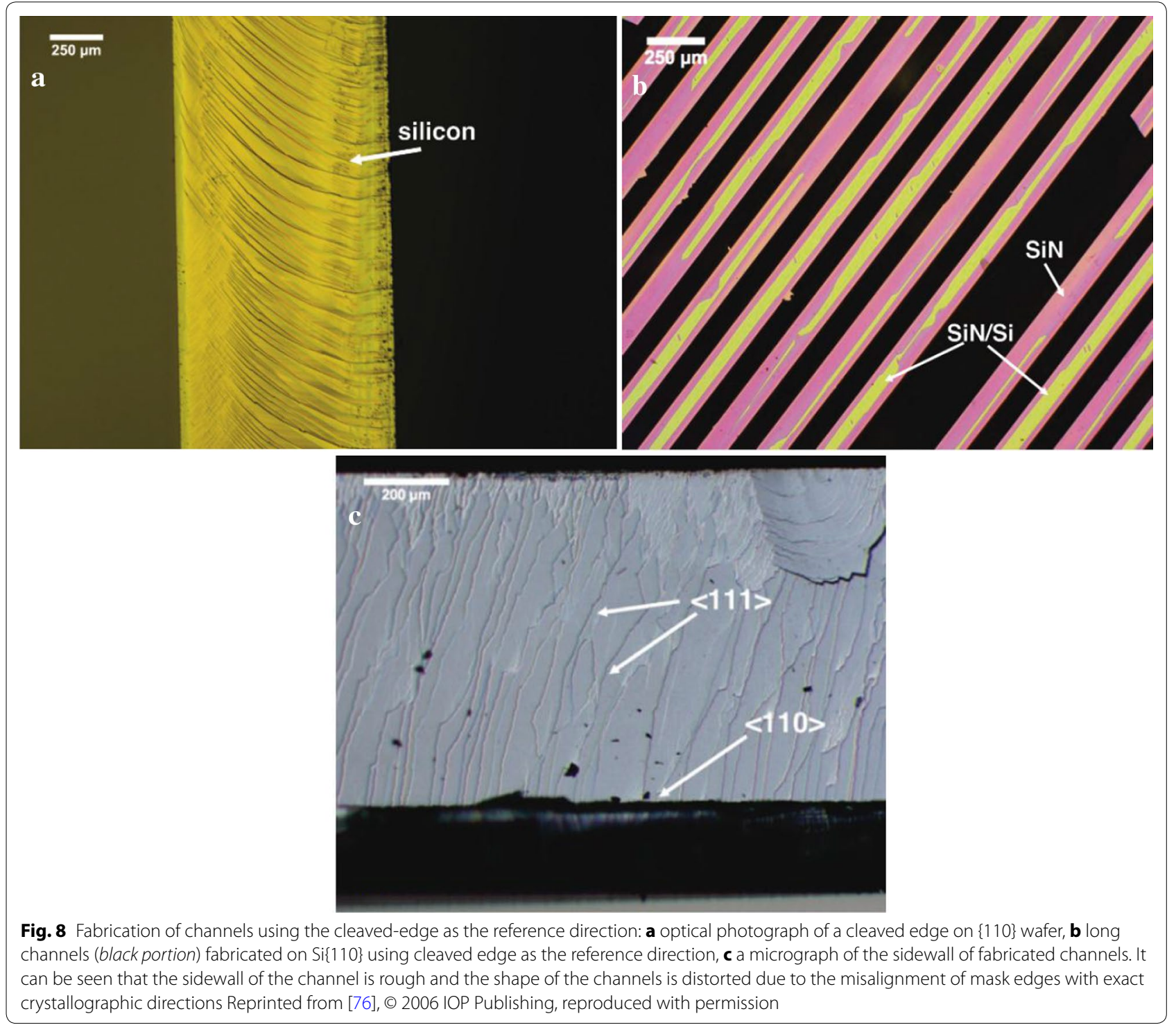

In order to determine the $\langle 110\rangle$ direction on $\mathrm{Si}\{100\}$ wafer, Ensell proposed a set of circular window feature as presented in Fig. 9 [104]. The etched pattern of circular windows results in square pyramidal structures enclosed by $\langle 110\rangle$ directions as shown in Fig. 9b. The number of circles in the pre-etched pattern depends on the accuracy of the wafer flat (i.e., $\langle 110\rangle$ direction). If the $\langle 110\rangle$ direction lies within $\pm 1^{\circ}$ of the wafer flat, the circular patters should cover an arc of $2^{\circ}$ on both side of the reference direction which is parallel to the wafer flat. At the same time, the diameter of the circles of the pre-etched patterns also depends on the accuracy with which the crystallographic direction needs to be identified. Although smaller circles will give better accuracy however it is difficult to investigate structures of small sizes under the mask aligner. Ensell fabricated the pre-etched pattern consisting of a series of 41 circular openings of diameter $75 \mu \mathrm{m}$ and spaced $78.5 \mu \mathrm{m}$ apart with an angular pitch of $0.1^{\circ}$ on an arc of radius $45 \mathrm{~mm}$. Forty-one circles require 20 circles on each side of the reference direction. Twenty circles with an angular pitch of $0.1^{\circ}$ correspond to $2^{\circ}$ on either side of the reference direction which is parallel to the flat. After patterning the geometry on diametrically opposite ends, etching is carried out in anisotropic etchant. Upon prolonged etching the circular openings takes the pyramidal shape as shown in Fig. 9b. The masking layer can be removed for better visualization. The misalignment of the edges of the neighbouring structures is used as a measure to determine the precise $\langle 110\rangle$ directions. As shown in Fig. 9b, as one move towards 


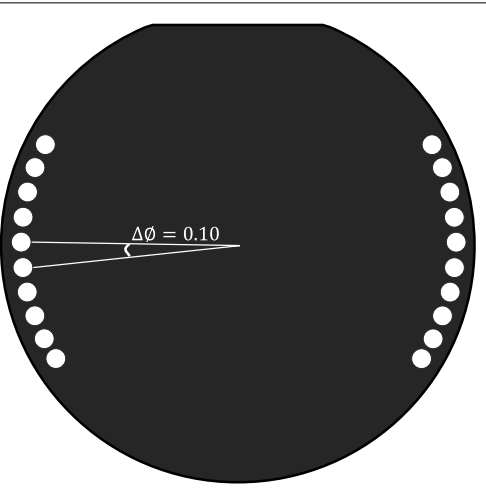

a

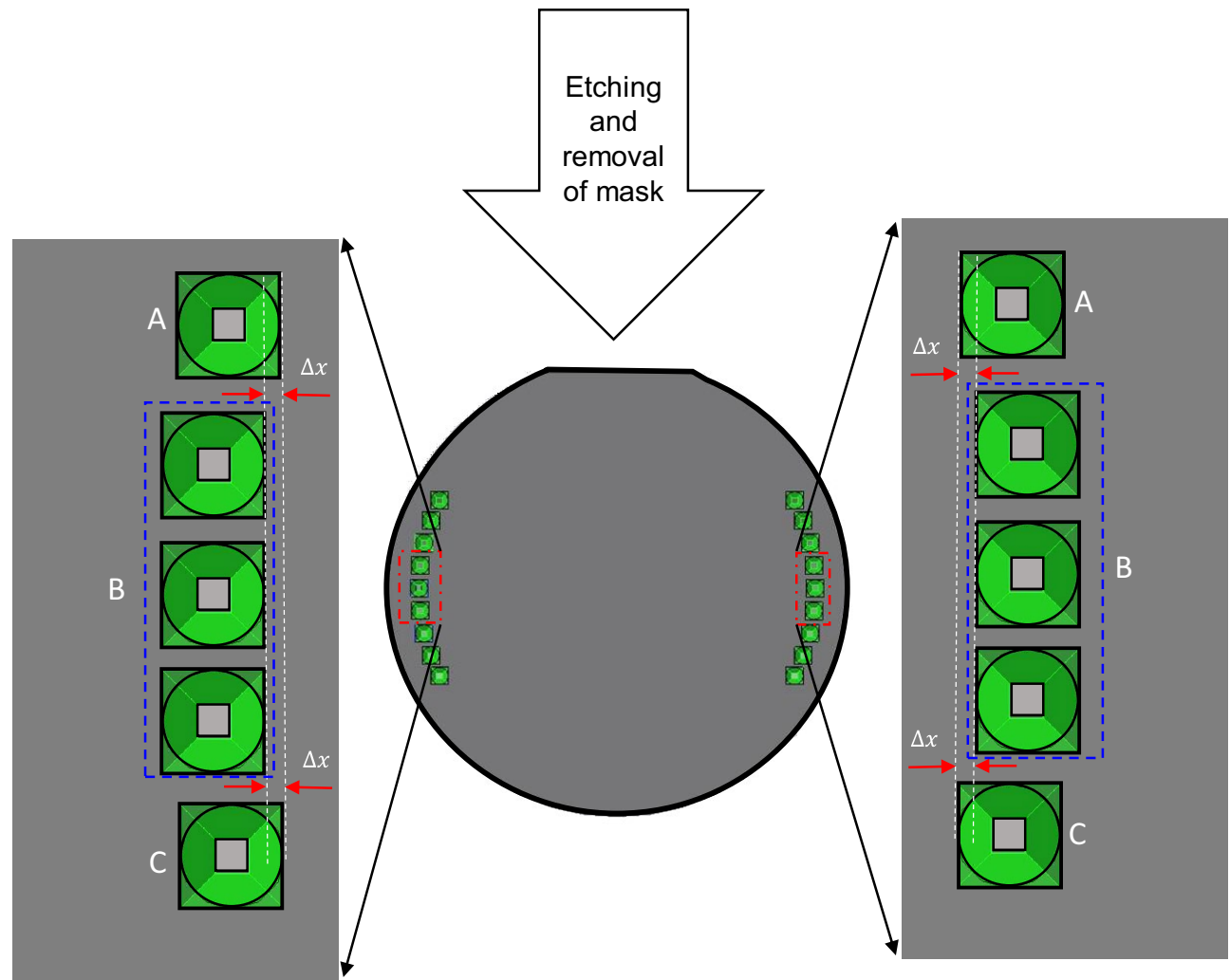

b

Fig. 9 Schematic representation of the technique proposed by Ensell: a circular openings patterned on an arc, $\mathbf{b}$ pyramidal shape etched patterns after anisotropic etching [104]

the centre from any end, the base of the pyramid shifts to one direction. Moving down from top (Structure A), one sees that structures $B$ is shifted (offset) by $\Delta x$. The structures in B however are approximately aligned in a straight line. Moving further down to structure $\mathrm{C}$, it can be seen that the base shift changes its direction. This implies that the precise $\langle 110\rangle$ direction lies between structure $\mathrm{A}$ and $\mathrm{C}$. If at all the shift $\Delta \mathrm{x}$ is same between $A$ to $B$ and $B$ to $C$, then the $\langle 110\rangle$ direction pass exactly through the central pyramid of structure $B$. This extent of offset is a measure of the misalignment with the $\langle 110\rangle$ direction. The author proposed to inspect the etched patterns to find the three pyramids (structures B) with the closest alignment of edges to determine the crystal direction. After selecting the three pyramids whose edges are almost exactly aligned to each other, the central pyramid is considered to be aligned along the precise $\langle 110\rangle$ direction. For an angular interval of $\Delta \emptyset=0.1^{\circ}$, the minimum value of $\Delta \mathrm{x}$ for the given dimension of the pre-etched patterns is $0.07 \mu \mathrm{m}$ and with every increase of $0.1^{\circ}$ in $\Delta \emptyset$, 
$\Delta \mathrm{x}$ increases by $0.14 \mu \mathrm{m}$. After finding the cavity across which the offset is minimum and changes its direction, the precise $\langle 110\rangle$ direction can be identified based on the value of $\Delta \mathrm{x}$ of the neighbouring cavity as explained above. In order to increase the accuracy, the dimensions of the circles can be reduced in which case there would be lesser number of pyramids whose edges align precisely. This is a very simple technique both in terms of design and its implementation in finding the crystal direction. The author visually inspected the structures to determine the pyramids with almost exactly aligned edges. However, this is not the most accurate methodology as the submicron offset is not visible under the microscope. For most accurate results, the offset $\Delta \mathrm{x}$ should be measured for each structure to determine the one with minimum offset which would correspond to the precise $\langle 110\rangle$ direction. However, this minimum offset length is of the order of submicron range which is difficult to measure on a normal mask aligner and thus it needs sophisticated equipment.

Vangbo and Backlund proposed an alignment fork as the pre-etched pattern to determine the $\langle 110\rangle$ directions
[102]. The property of symmetric undercutting across the $\langle 110\rangle$ direction is exploited to determine the $\langle 110\rangle$ directions. The pattern comprises of a set of three ridges tapered by small angles $(\alpha, \beta)$ as shown schematically in Fig. 10. This set of structure is then repeated at an angular interval of $0.1^{\circ}$. Using this pre-etched pattern, the $\langle 110\rangle$ direction can be determined using two effects. The first is the tapered ridge effect. Upon etching, the dimension of the ridge will start to increase where it is narrowest and finally will lead to an oversized opening. Smaller angle of tapering ensures faster etching time to form the final undercut structure. If the ridges happen to be lying symmetrically across the $\langle 110\rangle$ direction on the patterned wafer, then the undercut lengths of the two ridges will be same. In order to determine the $\langle 110\rangle$ direction, the etched patterns are carefully inspected to find the pair of ridge which is symmetrically etched. This is one method to determine the precise $\langle 110\rangle$ direction. Another method is by using the tapered groove effect. In this method, the mask pattern comprises of ridges which widen with a small angle. As etching progresses, the ridges takes the shape of grooves and the bottom

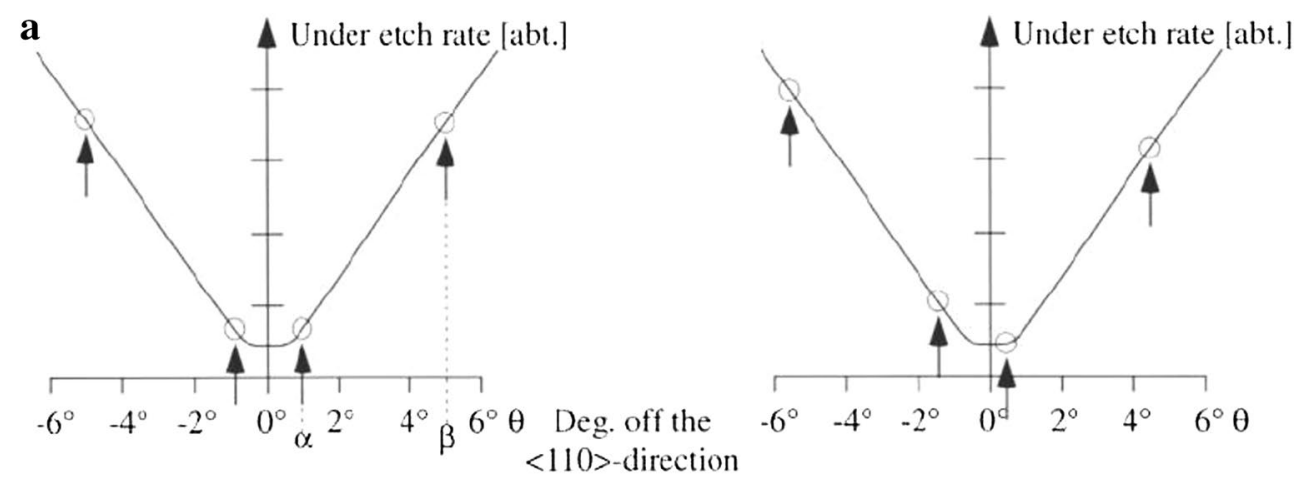

b

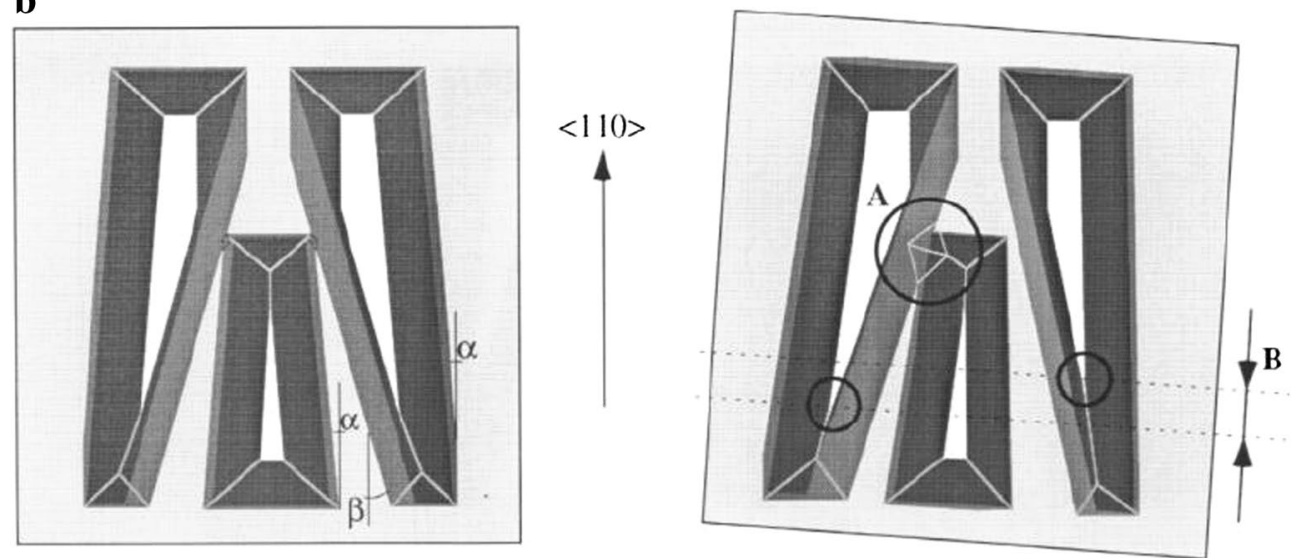

Fig. 10 A technique to determine the $\langle 110\rangle$ direction on Si\{100\} surface proposed by Vangbo and Backlund exploiting a the symmetric etch rate behaviour across the $\langle 110\rangle$ direction, and $\mathbf{b}$ the etched patterns comprising of ridges with tapered edges [102]. The set of ridges with symmetric undercutting constitutes the precise $\langle 110\rangle$ direction Reproduced with permission from [102]. ๑ 1996 IOP Publishing 
plane continues to diminish and the final etched profile comprises of intersecting $\{111\}$ planes. If the undercutting rate is low (i.e., near the $\langle 110\rangle$ direction), the grooves will be narrower and more complete (i.e., the base will have a longer intersection). However, if undercutting rate is more (i.e., away from $\langle 110\rangle$ direction) the groove will be wider with a longer length of incomplete portion of the base for the same etching time. In order to determine the correct $\langle 110\rangle$ direction, the grooves can be inspected under a microscope to determine and compare the lengths of the incomplete portion of the formed grooves. For alignment of subsequent mask edges, a square window is added corresponding to each alignment fork. The entire set is now repeated at an angular interval of $0.1^{\circ}$ with the centre point of rotation at the opposite side of the wafer. Additionally, a similar square is also patterned at the centre of rotation itself. Now, the subsequent mask is also added with two similar squares on each side. During subsequent alignment, one is overlapped with the square at the centre of rotation and the other is overlapped with the square corresponding to the fork where symmetric undercutting occurs. This is an easy method as the precise direction can be determined by a visual investigation of set of structures to find one with symmetric undercut. However, this can be a tedious task as the structures near the $\langle 110\rangle$ direction can have similar undercut which may not be distinguishable.
The source of error can be removed by measuring the undercut lengths to determine the symmetric undercut structures but it would require sophisticated equipment. At the same time, the varying undercut rate at different places on the wafer may also trick the human eye and give erroneous results. Similarly, determining the incomplete length of the base of the groove under a microscope will depend on the judgement of the user. This can lead to errors in determining the crystal direction.

Another method to determine the $\langle 110\rangle$ direction on $\mathrm{Si}\{100\}$ wafer was proposed by Lai et al. where they used a series of rectangular and squared openings stacked on an arc of radius $R$ which is greater than the diameter of the wafer [105]. These patterns were fabricated on one side of the wafer and a series of only square windows called auxiliary set on the diametrically opposite sides. These two set of patterns fabricated on opposite ends lie on an arc of concentric circles. The schematic diagram of the pre-etched patterns is shown in Fig. 11. The width of the squared geometry as well as the rectangular geometry is kept the same $(W)$ because the vertical distance between the edges of the square and rectangular pattern after etching is a measure of the extent of misalignment. On prolonged etching, the rectangular and square openings take the shape of inverted pyramid. Depending on the degree of misalignment of the pattern edges with the $\langle 110\rangle$ directions, the structures will become oversized.

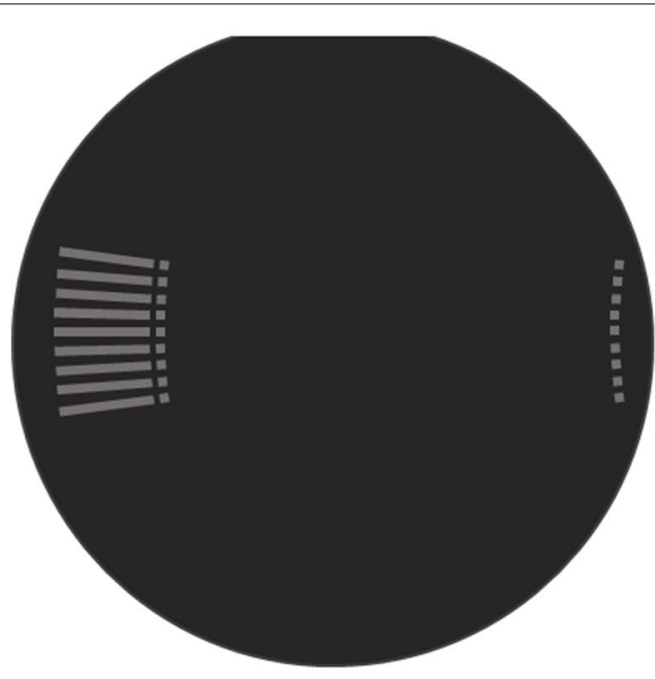

a

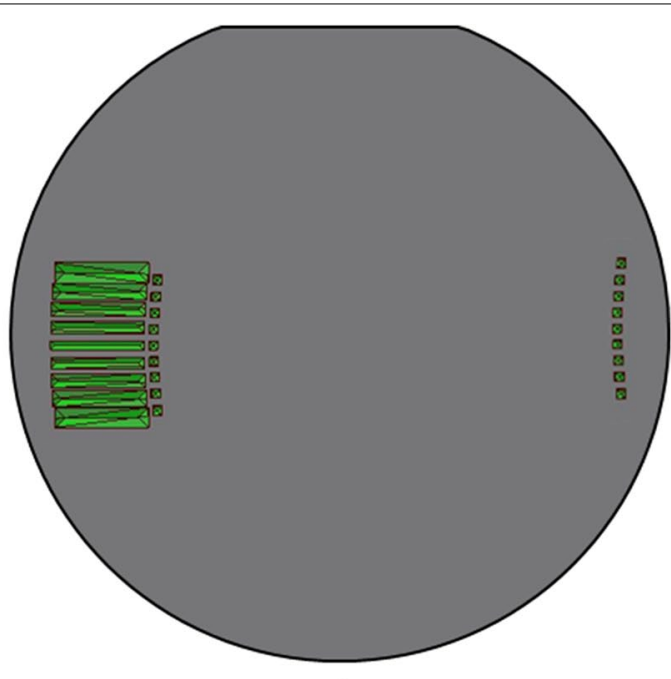

c

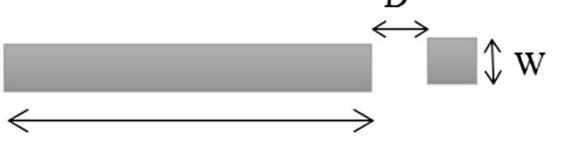

L

b

Fig. 11 Schematic diagram of the technique used by Lai et al.: a Patterning of pre-etched patterns on one side and an auxiliary set consisting of only square windows on the other side, $\mathbf{b}$ dimension of each pattern of the set, $\mathbf{c}$ etched profile after anisotropic etching [105] 
As a result, the difference in width of the rectangular and square opening (indicated by $\Delta y$ in Fig. 12) increases. Figure 12 shows the etched profile of the patterns on prolonged etching. From geometry $\Delta y$ can be calculated using the following relation

$$
\Delta \mathrm{y}=(\mathrm{L}+\mathrm{D}) \sin \delta
$$

At the precise $\langle 110\rangle$ direction, $\delta$ is zero, thus the vertical distances between the edge of square and rectangular geometry $(\Delta y)$ is almost zero. However, as we move away from the $\langle 110\rangle$ direction, the vertical distance keeps on increasing and finally merges in the case of large deviation from the $\langle 110\rangle$ direction as shown in Fig. 12. The pattern with minimum $\Delta \mathrm{y}$ is selected and aligned with dimensionally similar pattern on the subsequent mask. The advantage of this method is that the structures far away from the precise $\langle 110\rangle$ direction will get merged to each other, while the structures in the vicinity of $\langle 110\rangle$ direction will remain un-merged. This will reduce the domain of investigation to find out the precise $\langle 110\rangle$ direction. However from Eq. (3) we see that for a misalignment of $0.01^{\circ}$, the value of $\Delta y$ is $0.18 \mu \mathrm{m}$ when the rectangular opening is $1000 \mu \mathrm{m}$ long and the distance (D) between rectangle and square is $16 \mu \mathrm{m}$. Using an optical microscope the particular structure with this minimum undercut can be located to be corresponding to the precise $\langle 110\rangle$ direction. The next minimum undercut length for a misalignment of $0.02^{\circ}$ is $0.35 \mu \mathrm{m}$. As a result it should be ensured that the microscope is capable of distinguishing between these submicron distances. However, for precise identification, these undercut lengths can be measured to determine the structure with minimum undercutting. However, measurement of such small distances firstly requires sophisticated equipment and secondly it makes the task tedious. Nevertheless, the benefit of these patterns is that it provides a longer length for aligning the mask pattern at one side of the wafer; however at the other side of the wafer the aligning length is only $16 \mu \mathrm{m}$. This makes the technique prone to theta error as well. At the same time etching $1000 \times 15 \mu \mathrm{m}$ opening would require a considerable time. Although, the undercut length can be increased by increasing either $\mathrm{L}$ or $\mathrm{D}$ (or both) but this is generally not preferred because first it would significantly increase the etching time and second, it would occupy more wafer space. We can however reduce the theta error by patterning the pre-etched feature on the auxiliary set as well. This would provide a longer length on diametrically opposite sides of the wafer for subsequent mask alignment and thus would reduce the error. While the etching time in this case will remain

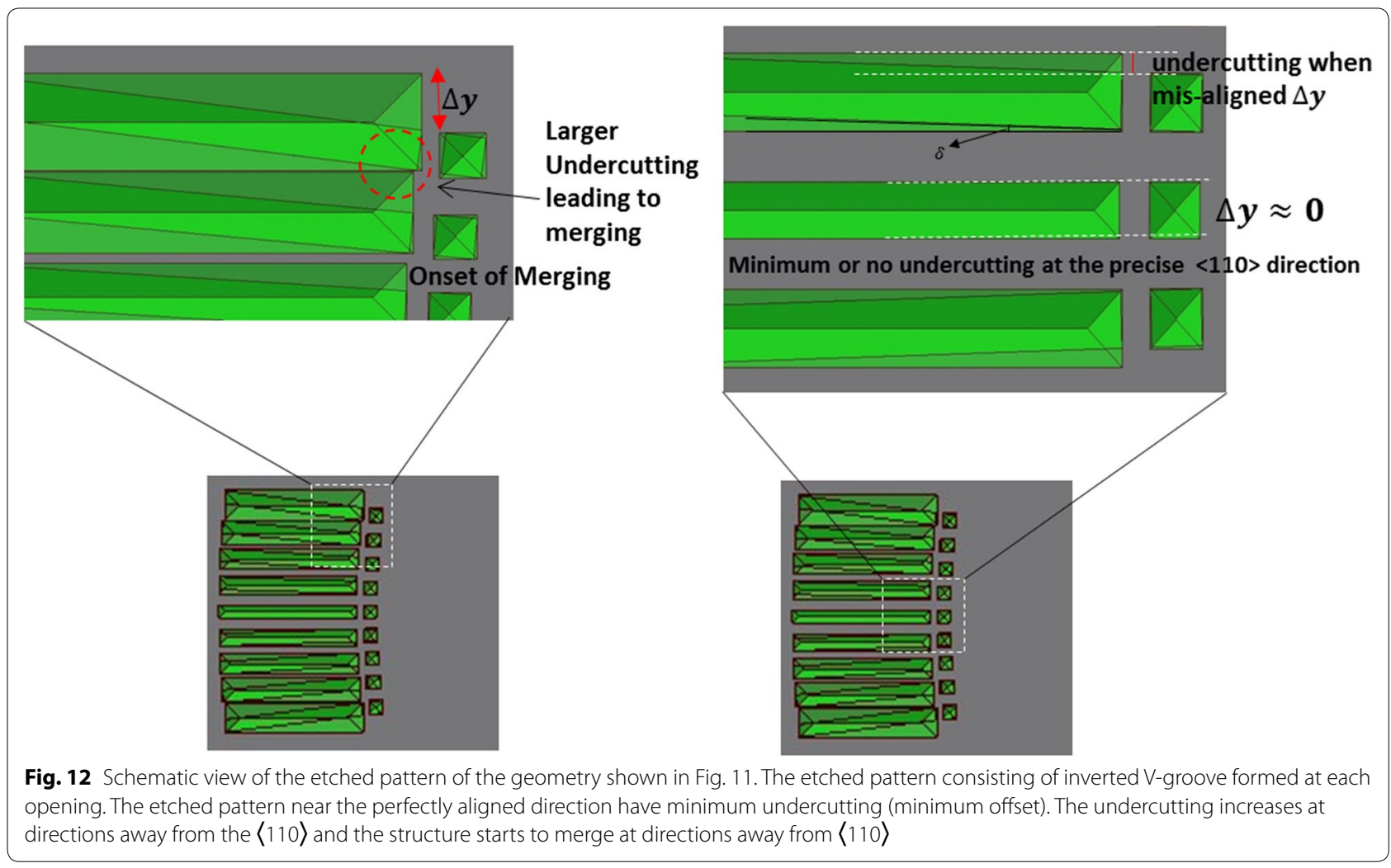


almost the same at in the previous case, but the usable space on the wafer is reduced.

In another study by Chang and Huang, a pre-etched pattern is utilized to determine the $\langle 110\rangle$ directions on both $\operatorname{Si}\{100\}$ and $\operatorname{Si}\{110\}$ wafer [107]. Additionally they also carried out the statistical analysis to calculate the precision of determination of crystal directions. Their analysis suggested that the precision with which the crystal orientation can be determined does not solely depend on the resolution of the pre-etching mask patterns but is limited by the finite step resolution of the mask generator. As a result they optimized the dimensions and the tilt angle so as to limit the error due to finite step of the pattern generator within the design's tolerance. However, here we discuss only the technique for precise determination of crystal direction. The proposed pre-etched pattern comprises of a series of trapezoids/ridge with the shorter sides parallel to each other and the longer sides inclined at different angles. The trapezoid with title angle of zero degree is designed to be parallel to the reference direction essentially the wafer flat ( $\langle 110\rangle$ direction). The dimensions of the height and the smaller edges of the parallel sides of each trapezoid is fixed as $\mathrm{H}=500 \mu \mathrm{m}$ and $\mathrm{W}=5 \mu \mathrm{m}$. The distance between consecutive trapezoids is also fixed as $\mathrm{D}=17.5 \mu \mathrm{m}$. A symmetric set is patterned across the reference line as shown schematically in Fig. 13. Upon etching, the trapezoidal opening takes the shape of rectangular grooves (Fig. 13b). The undercutting occurs due to misalignment of mask edges and the final structure is defined by $\{111\}$ planes. Due to the undercutting, the distance $\mathrm{D}$ reduces and finally becomes zero at a certain index T as shown in Fig. 13c. Similarly, other side of the reference direction also consists of a structure with index $\mathrm{T}^{\prime}$ where $\mathrm{D}$ becomes zero. In order to determine the misaligned angle, the following relation is used

$$
\psi_{M}=\Delta \psi\left(T-T^{\prime}\right) / 2
$$

where, $\Delta \psi$ is defined by $\psi_{\mathrm{i}}-\psi_{\mathrm{i}-1}$ which is $0.0125^{\circ}$. A positive value of $\psi_{M}$ implies that the crystal direction lies in the region of positive tilt angle $\psi_{i}$ while a negative value of $\psi_{M}$ implies that the crystal direction lies in the region of negative tilt angle $\psi_{i^{\prime}}$. In order to align the subsequent mask precisely along the crystal orientation, an alignment accessory pattern consisting of a series of circular opening is fabricated on an arc of radius $R$ with centre on the opposite side of the wafer where another circle is fabricated as shown in Fig. 14. The angular pitch of the circles on the alignment accessory pattern is the same as that of the preetched pattern. Additional structures called dial structures are also fabricated beside each ridge to indicate the index of the structure. After etching, the pre-etched pattern takes the shape of rectangular groove and the circles on the alignment accessory pattern as well as the circle at the other end of the wafer takes the shape of square cavity. Then, the index of the ridge corresponding to $\mathrm{T}$ and $\mathrm{T}^{\prime}$ as discussed earlier are obtained using the dial structure. Subsequently, the mis-orientation angle $\left(\psi_{M}\right)$ is calculated. Now, the square opening corresponding to this mis-orientation angle is selected from the alignment accessory pattern for subsequent alignment of mask. The subsequent mask which comprises of two squares: one for aligning the fabricated square on the other end of the wafer and the other for aligning the corresponding square from the alignment accessory pattern is then aligned as shown in Fig. 14. In cases where the wafer flat is perfectly aligned along the $\langle 110\rangle$ direction, the index of $\mathrm{T}$ and $\mathrm{T}^{\prime}$ will be same. This will make the mis-orientation angle $\psi_{M}=0$. The square opening corresponding to this mis-orientation angle on the alignment accessory pattern is the one beside the 0th ridge which is essentially parallel to the wafer flat.

This method can also be used to determine $\langle 110\rangle$ directions on $\mathrm{Si}\{110\}$ wafer. The etched geometry in case of $\mathrm{Si}\{110\}$ wafer is shown in Fig. 13d. This methodology does not require the measurement of small undercut lengths for precise determination of crystal directions. However distinguishing the target ridge from the set of etched ridge is a difficult task. At the same time the etching time increases due to long structures. Additionally it takes a lot of usable wafer space due to long length of the patterns which is itself complex in designing. Overall this methodology to determine the crystal direction is complicated as compared to several other simple methodologies which have been proposed.

The relative etch rates of silicon planes such as $\{100\}$ and $\{110\}$ can be altered significantly by the addition of a small amount of surfactant in high concentration TMAH (e.g. 20-25 wt\%) [1, 48-50, 111-119]. In surfactant added $25 \mathrm{wt} \% \mathrm{TMAH}$, the etch rate of $\{110\}$ planes is reduced to a considerably low level, while that of $\{100\}$ plane remains almost unaltered. This property of surfactant added TMAH is used to fabricate $45^{\circ}$ micromirrors which are used in optical MEMS to provide $90^{\circ}$ out-of-plane reflection as illustrated in Fig. 15 [120-122]. In order to ensure that the reflected beam is exactly perpendicular to the incoming beam, it is vital to ensure that the mirror walls are precisely at an angle of $45^{\circ}$. On $\mathrm{Si}\{001\}$ wafer, $\{011\}$ plane oriented at an angle of $45^{\circ}$ to the wafer surface appear at the $\langle 100\rangle$ direction. In order to achieve smooth and exactly $45^{\circ}$ sidewall, the mask edges must be precisely aligned along $\langle 100\rangle$ direction on $\mathrm{Si}\{001\}$ surface. Earlier attempt at determining the $\langle 100\rangle$ direction on $\mathrm{Si}\{100\}$ wafer was done by Chen et al. [108]. They exploited the phenomenon of undercutting at the contact line of the masking layer and the silicon substrate as a tool to determine the crystallographic directions. 

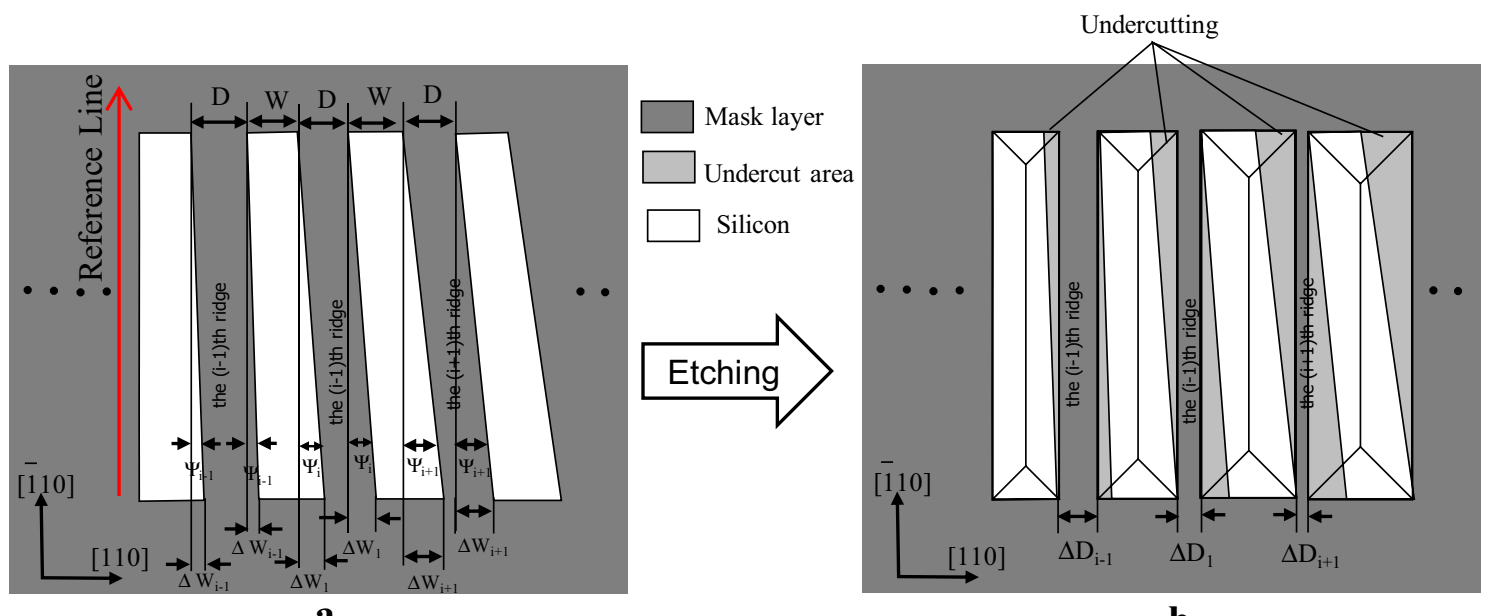

a

b

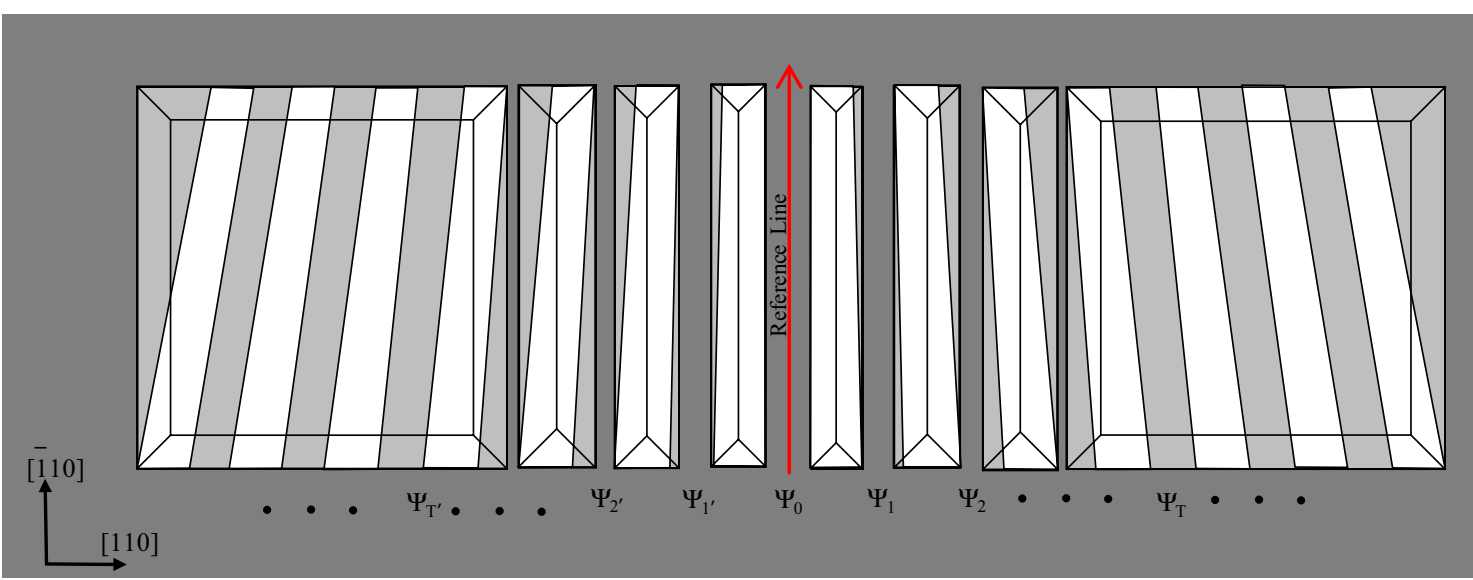

c

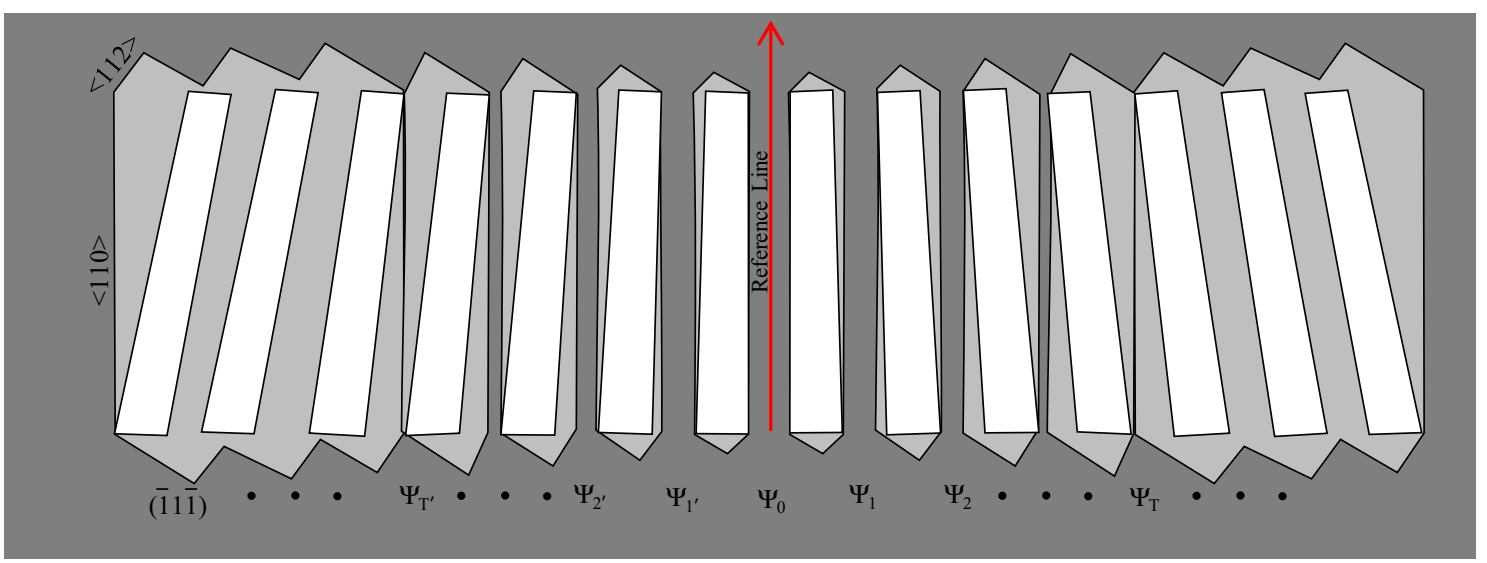

d

Fig. 13 Schematic representation of the technique using pre-etched pattern with tapered ridges as proposed by Chang and Huang: a A part of the patterned structures, $\mathbf{b}$ etched profile on Si\{100\}, $\mathbf{c}$ the entire etched patterns on Si\{100\}, d etched profile on Si\{110\} [107]. The index of ridge with $D=0$ (indexed by $T$ and $T$ ) on both sides of the reference line is used to determine the misalignment Reprinted from [107], $\odot$ 2005 Springer, reproduced with permission 


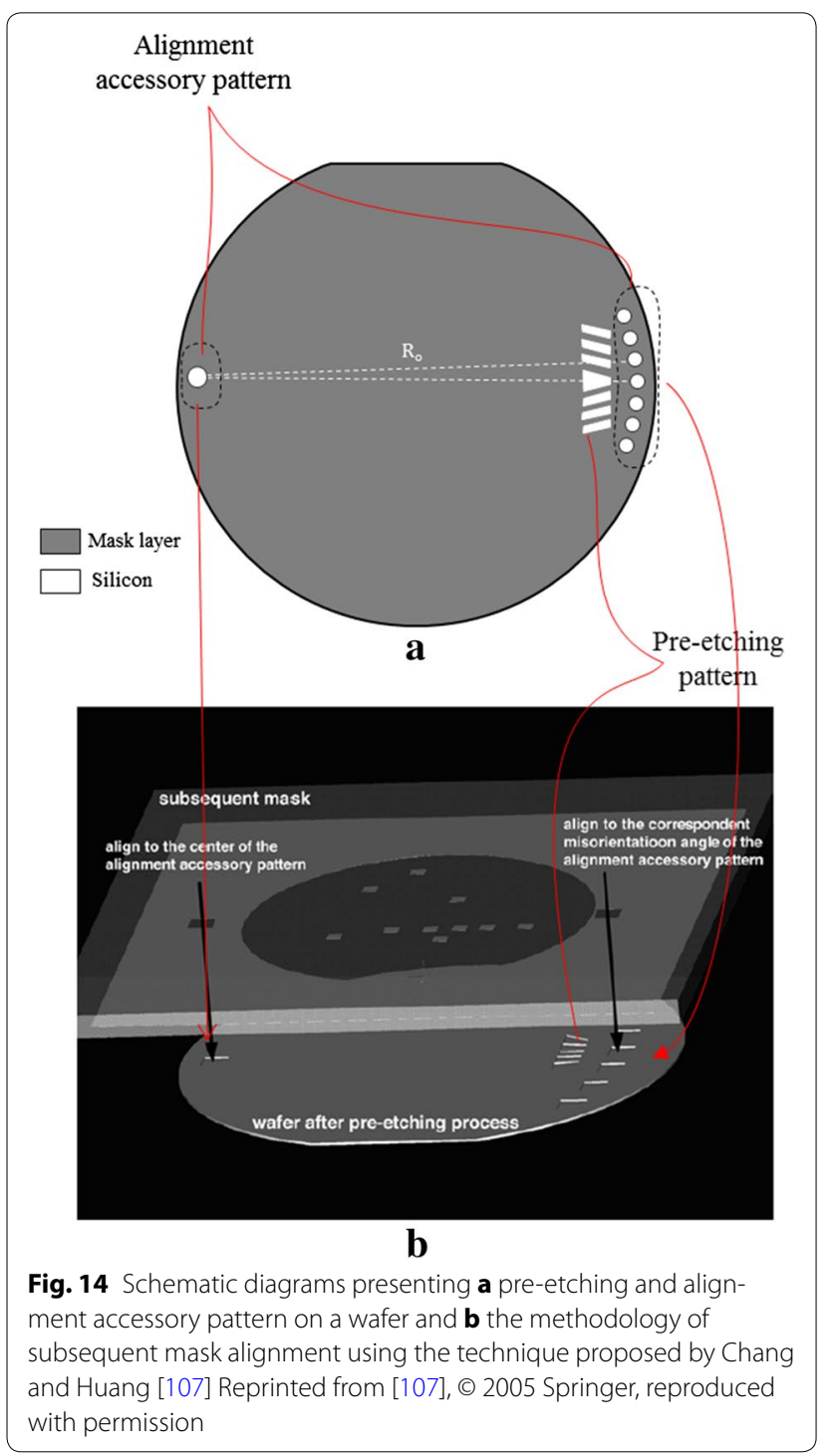

The pre-etched pattern comprises of 21 circles of $50 \mu \mathrm{m}$ diameter fabricated on an arc of $43 \mathrm{~mm}$. The angular pitch of the circles is $0.1^{\circ}$. Figure 16 shows the schematic diagram of the proposed patterns. Upon etching, the circular opening starts to distort. As the etching continues further the corners of adjacent squares tends to merge with each other. The authors observed the number of such merging to determine the precise direction. In the

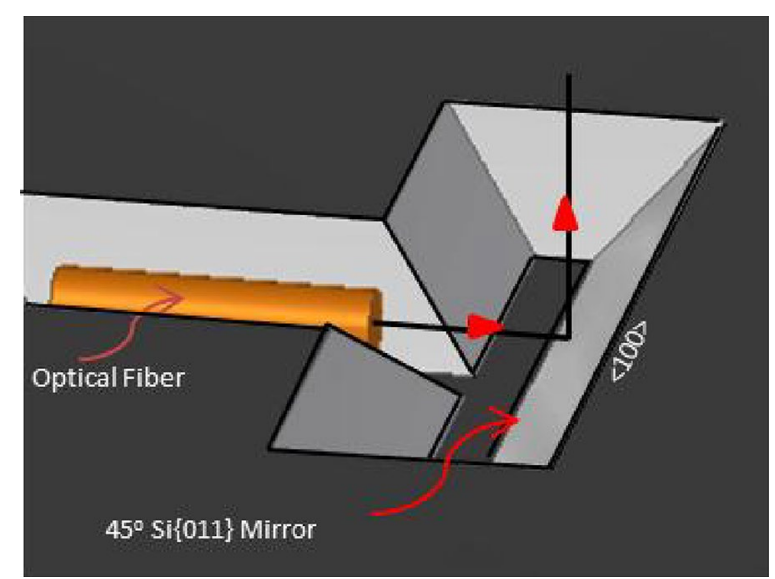

Fig. 15 Schematic diagram showing the use of Si\{110\} planes (at $\langle 100\rangle$ direction) slanted at an angle of $45^{\circ}$ to the wafer surface as micromirrors. The incoming beam is reflected $90^{\circ}$ out of plane Reproduced with permission from [110], (c 2016 IOP Publishing

cases where there is only one merging point, the precise $\langle 100\rangle$ direction lies through the merged point. If the number of merged corners is even, then the precise direction passes through the center of the central square and if the number of merged structures is odd, then the $\langle 100\rangle$ direction passes through the central merged point. In this technique, the number of merged points may depend on the etching time. This technique does not require any measurement to determine the undercut lengths and the location of the precise direction can be done by simply observing the number of merging of corners. However, this method is correct under the assumption that the undercutting is uniform for all the structures on the wafer. In practical applications, the undercutting varies for structure to structure even on the same wafer [123, 124]. As a result the reproducibility and correctness of this methodology is questionable as the number of merging can vary depending on the etch rate and can lead to error in the determination of precise crystallographic directions. At the same time, for subsequent alignment, one has to orient the wafer so as to align the mask edge to either small corner points or diagonal of the formed square. Aligning along both these is a tough task as well as prone to error owing to their small size. 


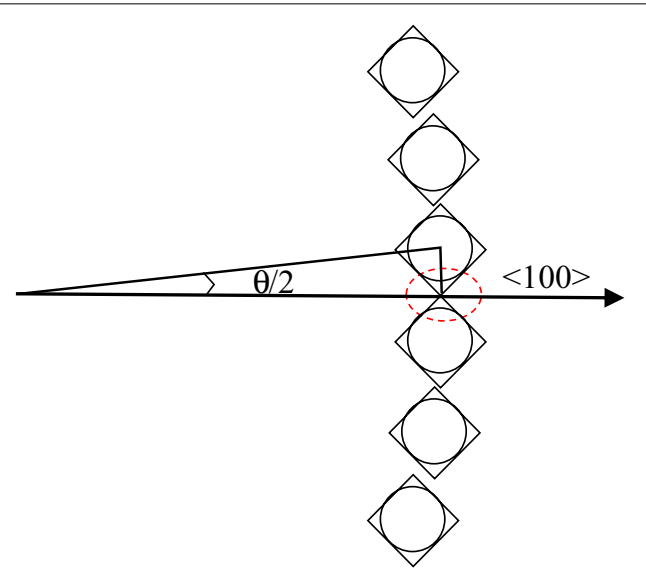

a

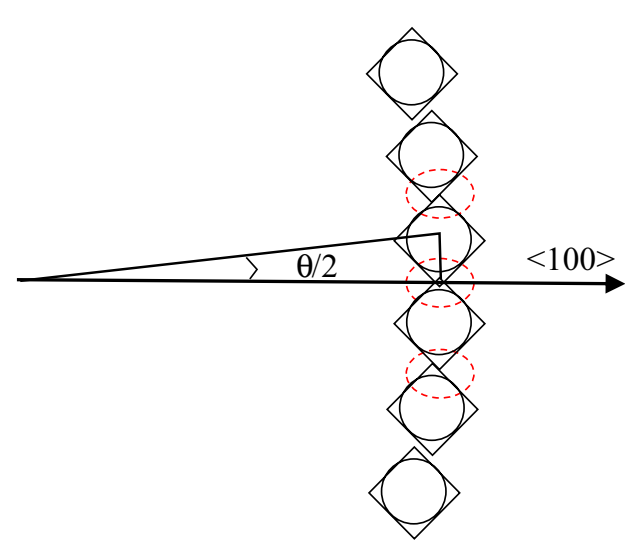

b

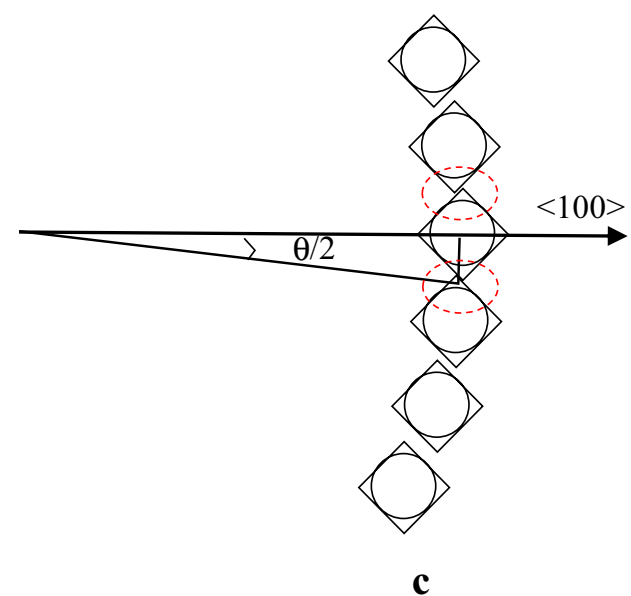

Fig. 16 Schematic representation of the technique comprising of circular openings suggested by Chen et al. [108]. After etching, due to underetching at the mask edges, the corners of the square merge with each other with $\mathbf{a}$ just one corner merging, $\mathbf{b}$ odd number of corners merging $\mathbf{c}$ even number of corners merging. In the first case, the $\langle 100\rangle$ direction lies between the two merged corners. In the case of odd number of merging, the precise $\langle 100\rangle$ direction passes through the middle corner. In cases where there is even number of merges of corners, the $\langle 100\rangle$ direction passes through the radial diagonal of the central square Reprinted from [108], @ 2000 SPIE, reproduced with permission

Recently, Sajal et al. proposed a self-aligning technique to precisely identify the $\langle 100\rangle$ directions on $\mathrm{Si}\{100\}$ wafer [109]. The pre-etched pattern comprises of four circles of $100 \mu \mathrm{m}$ diameter each, fabricated with a spacing of 43.5 , 44.5 and $45.5 \mu \mathrm{m}$. This differential spacing is incorporated in order to overcome the possibility of merging of notching due to overetching. The set of four circles is repeated at an angular interval of $0.17^{\circ}$ on both side of the reference line which is at an angle of $45^{\circ}$ to the wafer flat as shown in Fig. 17. The center of all the four circles lies on a straight line passing through the center of the wafer. The similar set of geometry is also patterned on diametrically opposite end of the wafer to reduce the theta error while subsequent aligning. The patterns are etched in an anisotropic etchant and the circular opening takes the shape of square V-groove with $\{111\}$ sidewalls as explained in the "Background" section. The optical image of the etched pattern is shown in Fig. 18. Near the perfectly aligned 

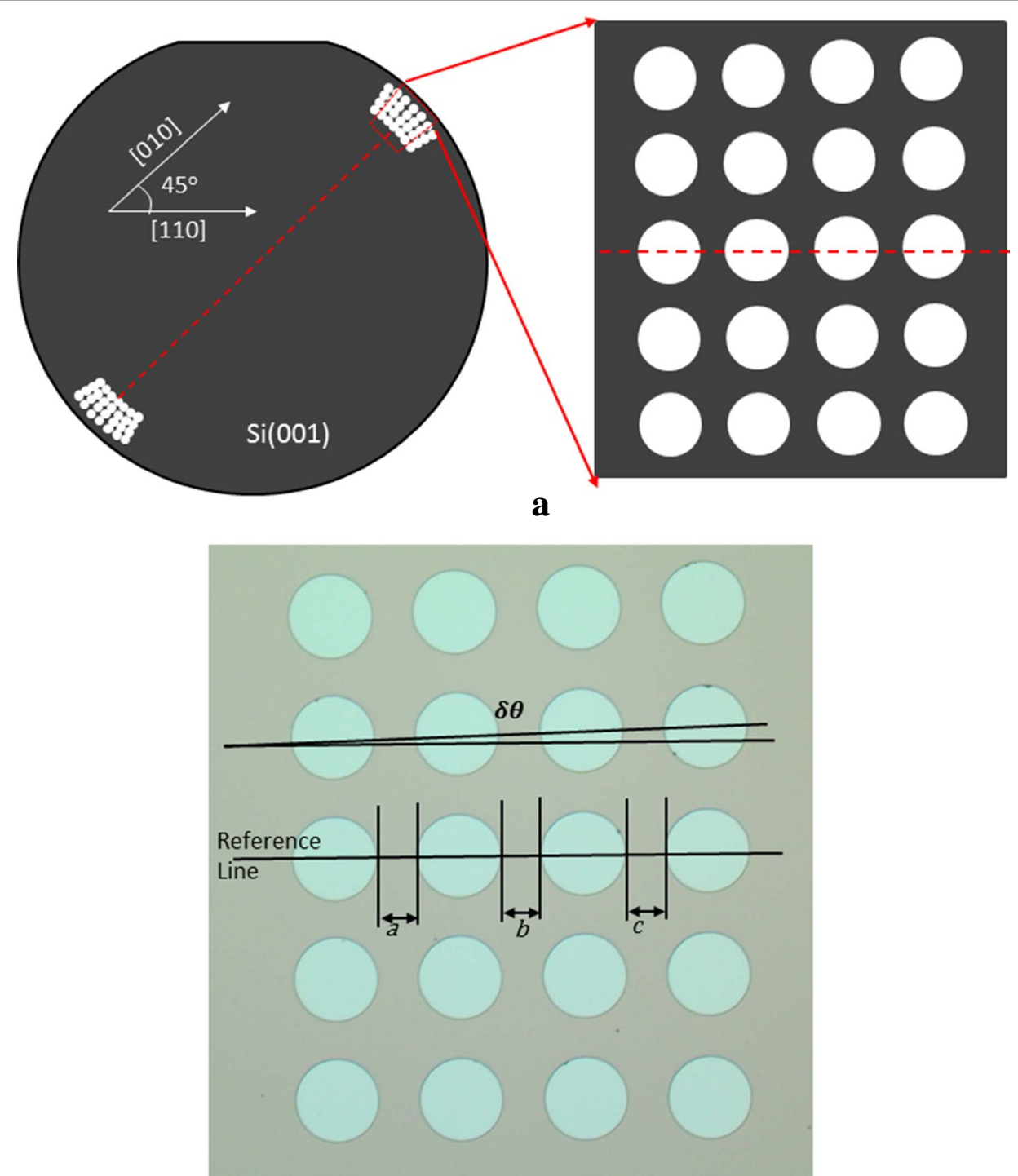

b

\begin{tabular}{|c|c|}
\hline Quantity & Value \\
\hline $\begin{array}{c}\text { Number of circles on } \\
\text { each side of wafer }\end{array}$ & $4^{*} 49=196$ \\
\hline Diameter of each circle & $100 \mu \mathrm{m}$ \\
\hline$(\mathrm{a}, \mathrm{b}, \mathrm{c})$ & $(43.5,44.5,45.5) \mu \mathrm{m}$ \\
\hline$\delta \theta$ & $0.17^{0}$ \\
\hline
\end{tabular}

Fig. 17 The patterns comprising of circular opening proposed by Sajal et al. for identifying the $\langle 100\rangle$ direction on Si\{100\}: a schematic diagram b optical images of the arrangement of pre-etched pattern on diametrically opposite end of the wafer [109]. Table lists the details of the patterns Reproduced with permission from [110], @ 2016 IOP Publishing 


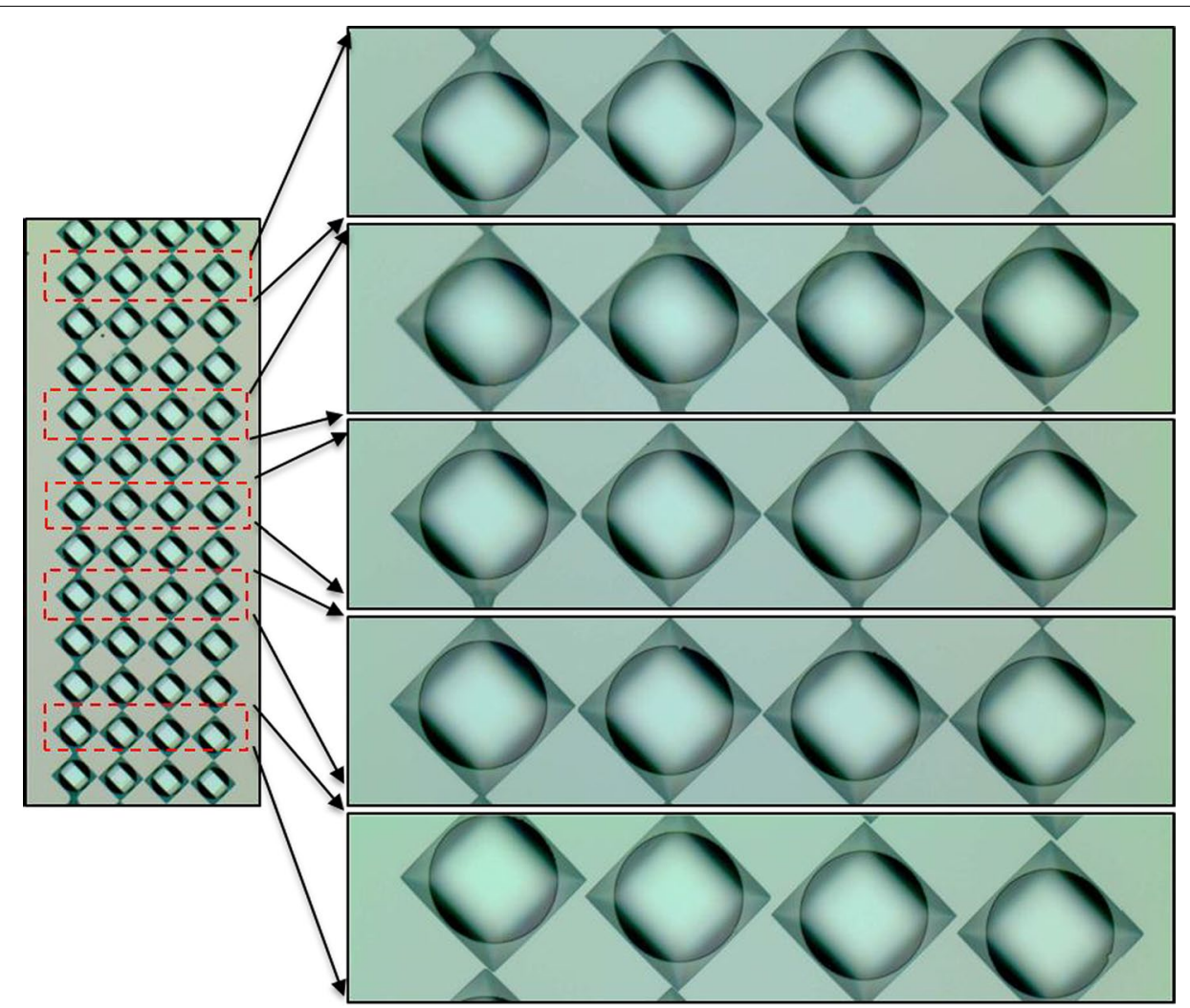

Fig. 18 Optical image of the etched profile of the pattern shown in Fig. 17 at two different magnifications. At the precise $\langle 100\rangle$ direction (centre pattern), the notches self-aligns itself to each other making it easily distinguishable with visual inspection. Moving away from the precise direction, the misalignment of the notches increases. This self-aligning features makes the precise $\langle 100\rangle$ direction obvious and inhibits the need of measurement of any kind Reproduced with permission from [110], @ 2016 IOP Publishing

$\langle 100\rangle$ direction, the radial diagonal of all the four squares lies on a straight line and the notch of the squares align perfectly to each other. As one move away from the precise $\langle 100\rangle$ direction, the notches starts to misalign and are no longer aligned to each other in a straight line as shown in Fig. 18. The extent of misalignment depends on the actual deviation from the precise $\langle 100\rangle$ direction. The misalignment of the notches also changes its direction across the $\langle 100\rangle$ direction, this also reduced the domain over which one needs to do a careful inspection to find the precise direction. As a result, with a visual inspection under a simple microscope the precise direction can be located without any need of measuring the undercut length. Thus, this is a measurement free technique to determine the crystallographic directions. The aligning of subsequent mask can be done by pre-fabricating a thin line at $45^{\circ}$ to the $\langle 110\rangle$ direction on diametrically opposite ends of the subsequent mask. This line can then be aligned along the self-aligned patterns which constitutes the $\langle 100\rangle$ direction on both sides of the wafer. The set of

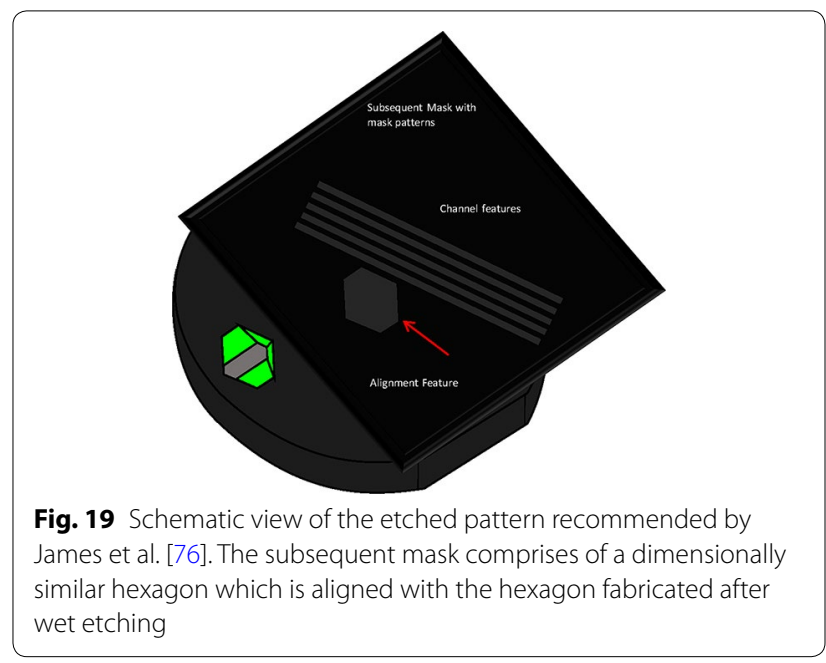

four circles which provides a longer length for the subsequent alignment of mask edges ensures reduction in the theta error. 

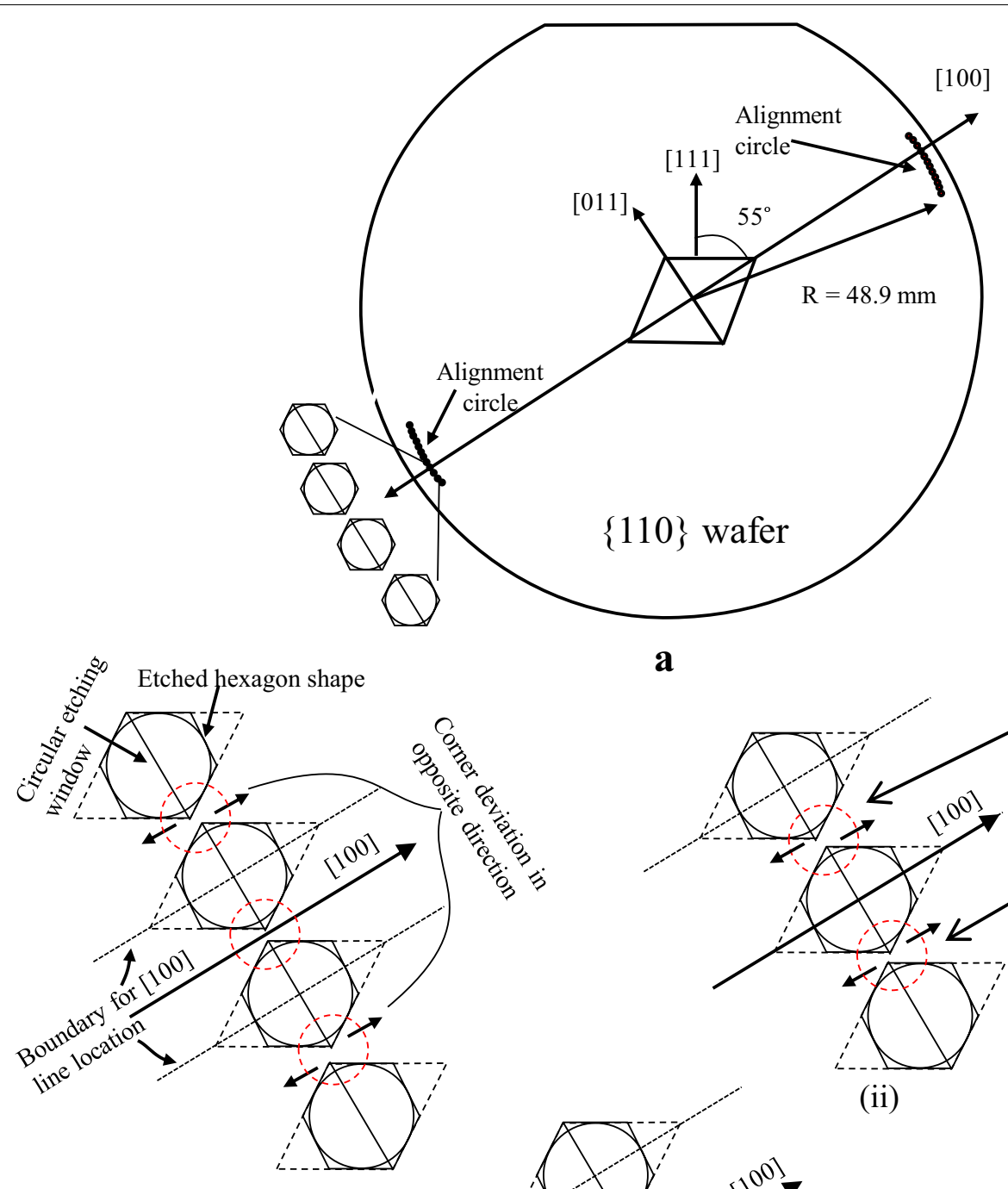

$\mathbf{a}$

(i)

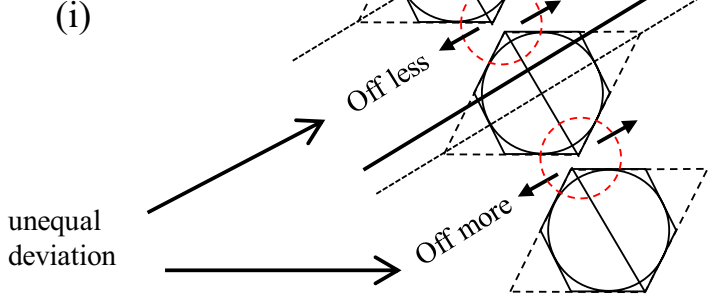

(iii)

Fig. 20 The pre-etched pattern proposed by Tseng and Chang showing the a location of the patterns and the deviation of the adjacent corners of the etched profile when there is $\mathbf{i}$ no deviation, ii equal deviation in opposite direction and iii unequal deviation in opposite direction [103]. In case of no deviation, the $\langle 100\rangle$ directions passes through the middle of the corners of the hexagons. In case of equal deviation, the $\langle 100\rangle$ direction passes through the center of the hexagon. In case of unequal deviation, the $\langle 100\rangle$ direction passes in between the two corners depending on the magnitude of the deviation Reprinted from [103], ๑ 2002 IOP Publishing, reproduced with permission 

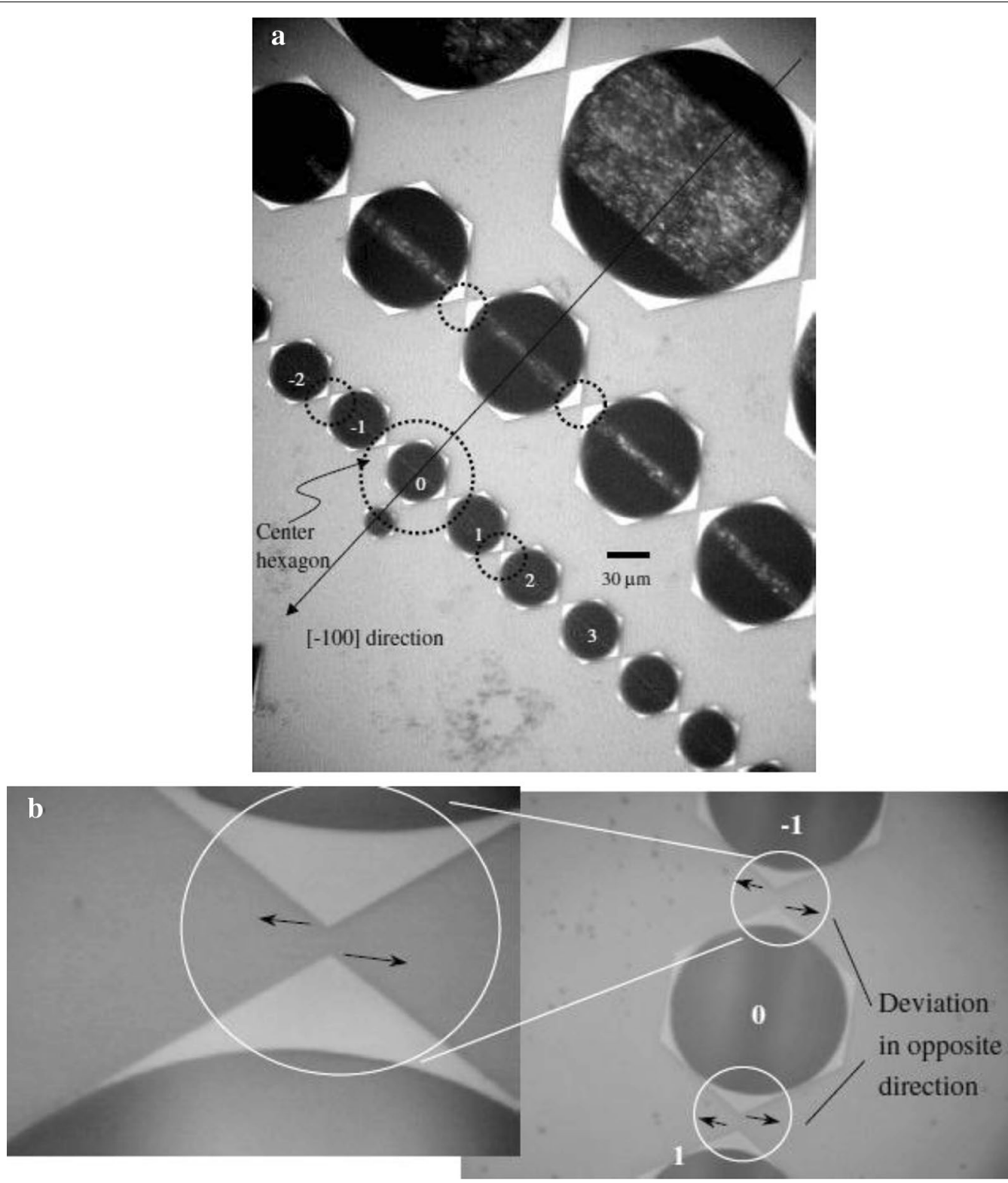

Fig. 21 SEM image of the etched profile corresponding to the patterns as shown in Fig. 20. a Circles takes the form of hexagonal grooves, b zoomed image showing the deviation of corners of adjacent hexagons Reprinted from [103], @ 2002 IOP Publishing, reproduced with permission 


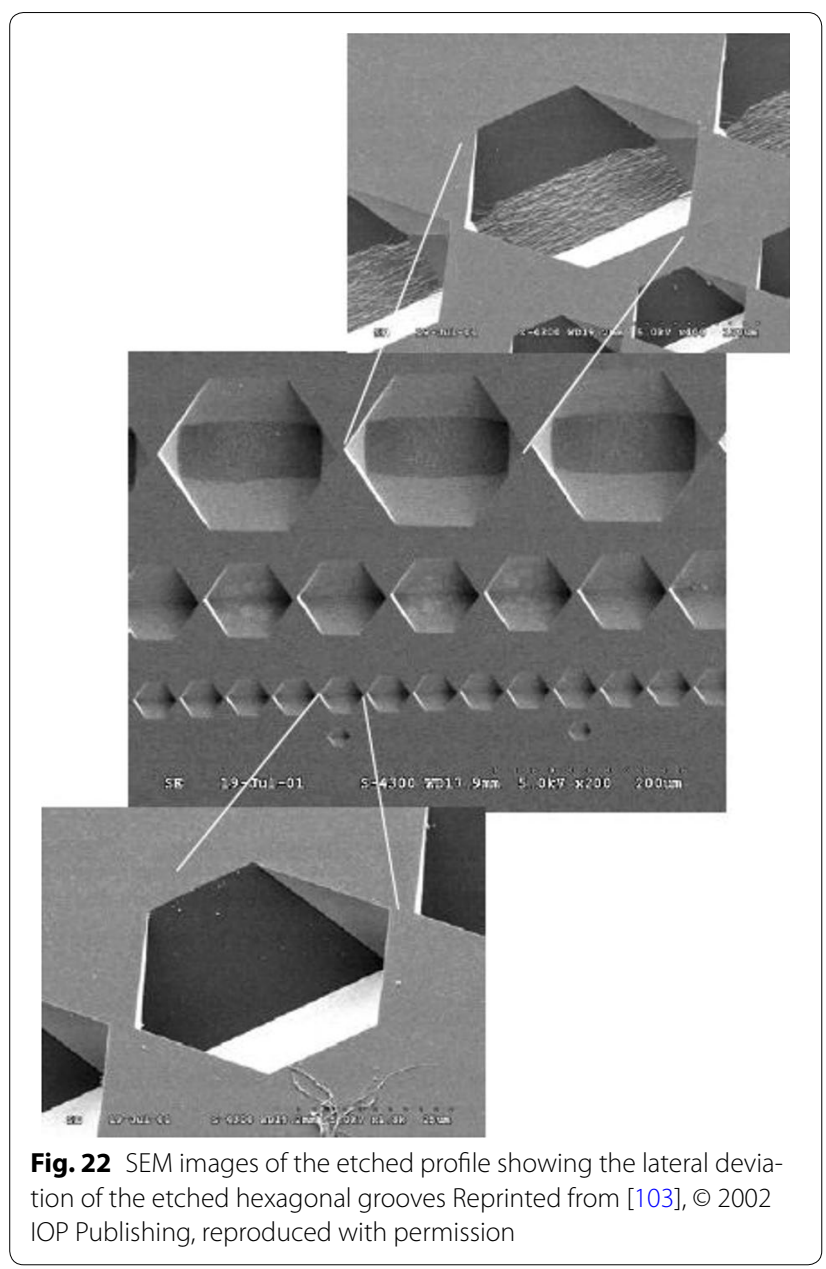

\section{Determination of crystal directions on Si\{110\} wafer}

On $\mathrm{Si}\{110\}$ wafer there are primarily two principal directions namely the $\langle 110\rangle$ and $\langle 112\rangle$ directions, which are useful owing to the appearance of slanted and vertical $\{111\}$ planes at these two directions, respectively. The appearance of vertical $\{111\}$ sidewalls makes $\mathrm{Si}\{110\}$ wafer indispensable to fabricate high aspect ratio microchannels and gratings using wet anisotropic etching for different applications $[19,76]$. There have been a few attempts to identify the above mentioned principal directions on $\mathrm{Si}\{110\}$ wafer.

In earlier attempts to precisely identify the $\langle 110\rangle$ direction, Ciarlo fabricated a fan like structure with rectangular openings of sides $8 \mu \mathrm{m}$ by $3 \mathrm{~mm}$ with longer length parallel to the wafer flat ( $\langle 110\rangle$ direction) [106]. This structure is then repeated at an angular interval of $0.1^{\circ}$. The obtained pattern is similar to that as shown in Fig. 11, however without the square adjacent to each rectangle. Prolonged etching leads to undercutting at the mask edges due to the misalignment of the edges with the $\langle 110\rangle$ direction. The structure with the minimum undercut would be the least misaligned with the precise $\langle 110\rangle$ direction. This method again deals with the disadvantage of selecting structures with small undercut lengths and increased etching time and using more wafer space etc.

In another method, James et al. used just one circle of diameter $1 \mathrm{~mm}$ as pre-etched pattern, which formed a hexahedron on prolonged etching [76]. The subsequent mask comprising of the structures to be fabricated also comprised of a dimensionally similar hexagon (alignment feature) which was formed after etching. The edges of the hexagon on the mask were then aligned along the edges of the hexahedron formed by wet-etching on the wafer as shown in Fig. 19. This way all the other structures to be fabricated could get aligned to the correct crystallographic direction. This method is also a very simple method to determine the $\langle 110\rangle$ and $\langle 112\rangle$ directions (given by the sides of the hexahedron) and it also requires less etching and processing time. However it again has the disadvantage of relying on aligning only along one side of the wafer making it prone to theta error.

Tseng and Chang attempted to determine the $\langle 100\rangle$ directions on $\mathrm{Si}\{110\}$ wafer employing the hexagonal shape of a circular openings obtained after etching as illustrated in Fig. 20 [103]. In their technique, they fabricated circular openings of diameter 153, 74 and $35 \mu \mathrm{m}$ with centre to centre distance of 192, 96 and $48 \mu \mathrm{m}$ on an arc of radius $48.9 \mathrm{~mm}$ at 55 and $-125^{\circ}$ to the $\langle 111\rangle$ directions. After etching, all the circular openings take the shape of hexagonal groove as explained in the "Background" section. Figures 21 and 22 show the SEM images of the fabricated structures. From the fabricated structures, that particular hexagonal structure is selected across which the direction of lateral deviation of corners changes its direction. The relative positions of the corners of the neighbouring hexagons to this hexagon is used to determine the precise $\langle 100\rangle$ direction. Figure 20 depicts schematically the top view of the etched profile of hexagonal grooves. It can be seen that there are three possible cases of the orientation of the corners of the adjacent hexagons. One of the possible orientation is that the corners of adjacent hexagons are precisely aligned to each other and the neighbouring 
a

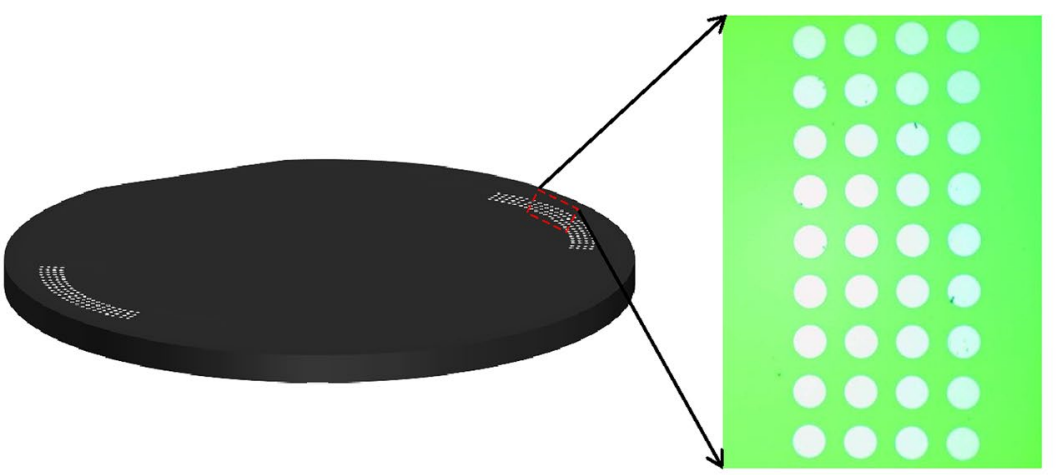

b

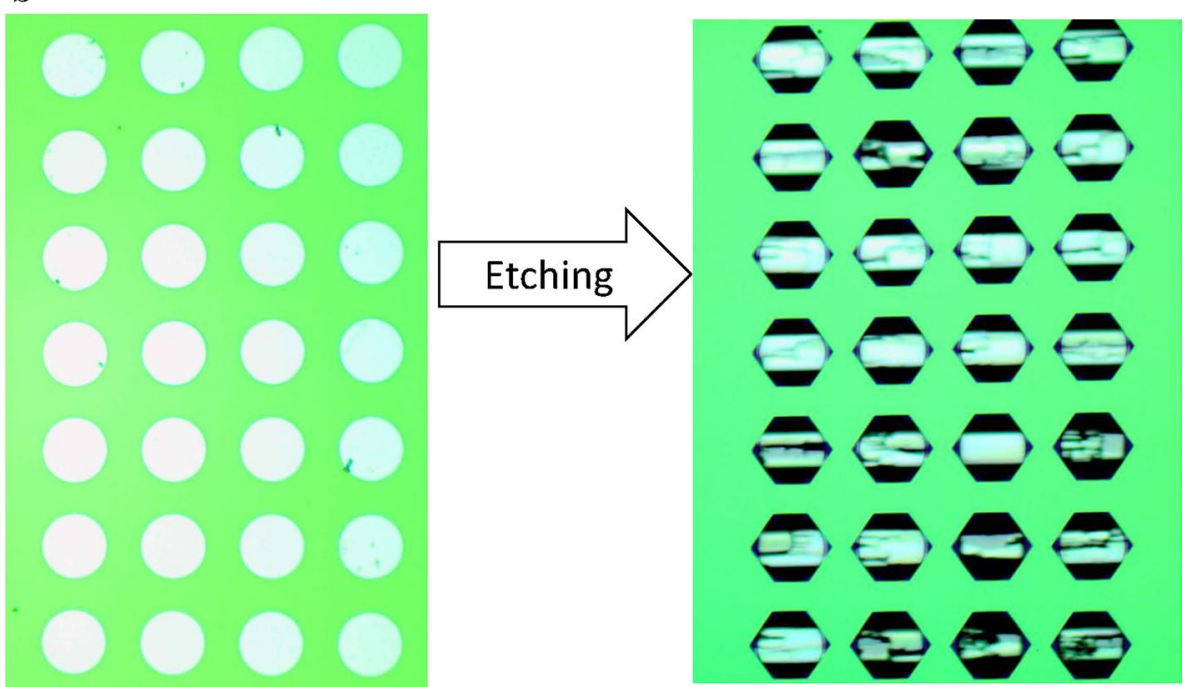

Fig. 23 The pre-etched pattern consisting of circular openings proposed by Sajal et al.: a schematic view showing the location and the optical image of the pattern, $\mathbf{b}$ after etching the circular opening takes the shape of hexagonal grooves [110]

hexagons have corners deviated in opposite directions. In this case the precise $\langle 100\rangle$ direction will lie perfectly in between the corners of the two aligning hexagons as presented in Fig. 20i. The second case is that the corners of hexagons on either side are deviated in opposite directions but with equal magnitude. In this case the precise $\langle 100\rangle$ direction will pass exactly through the centre of the hexagon as illustrated in Fig. 20ii. The third case is the intermediate case where the precise $\langle 100\rangle$ direction will pass through a point in between the two cases mentioned above depending on the magnitude of lateral deviation as shown in Fig. 20iii. Using this technique, the $\langle 100\rangle$ directions on $\operatorname{Si}\{110\}$ wafer can be determined. This technique has the advantage of being measurement free. However for subsequent alignment, one has to orient the wafer so as to align the mask edge with respect to two corner points unlike long edges in other cases. This task becomes even more difficult when the $35 \mu \mathrm{m}$ circles are used as reference for better accuracy where high magnification microscope is required to see the deviation. Other circles of larger diameter help in reducing the domain of investigation. However, it increases the etching time and occupies more usable space.

Recently, Sajal et al. presented an improvised measurement free technique to determine the $\langle 110\rangle$ direction on $\operatorname{Si}\{110\}$ wafer [110]. A series of circular openings of diameter $100 \mu \mathrm{m}$ were fabricated on four concentric arcs. Upon etching the circular openings took the shape of hexagonal grooves as shown in the optical image in Fig. 23. 


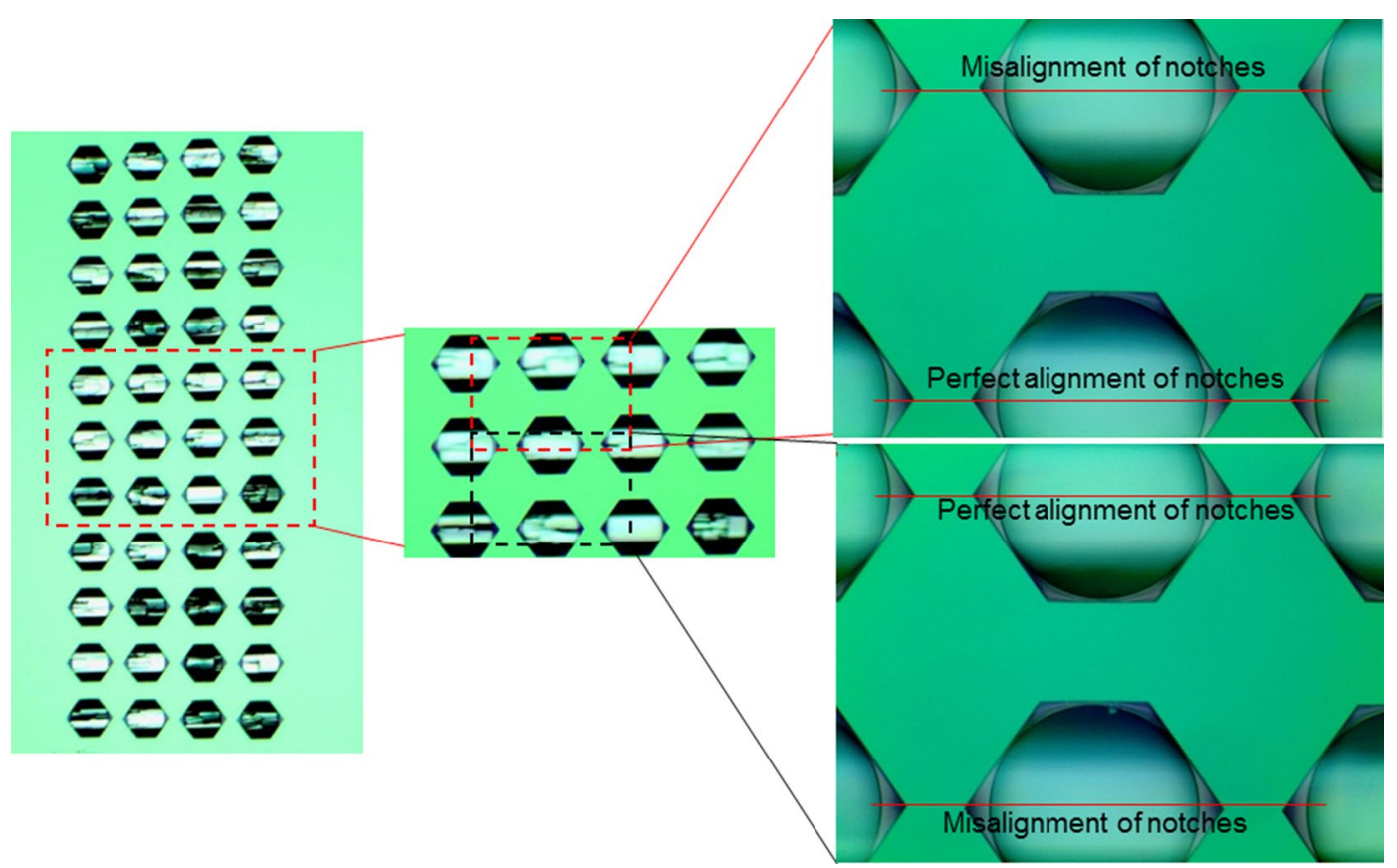

Fig. 24 Optical images of the etched patterns of a technique presented in Fig. 23 showing the self-aligning nature of the structures zoomed at different magnifications. At the precise $\langle 110\rangle$ direction, the notches of the four hexagons aligns exactly to each other while getting misaligned at directions away from $\langle 110\rangle$. The central pattern exhibits the precise alignment of notches. At the same time, the immediately adjacent patterns (both top and bottom) can be seen misaligned. As a result the $\langle 110\rangle$ direction appears obvious

Near the precise $\langle 110\rangle$ direction, the notches of all the four radial hexagons aligns to each other in a straight line while getting misaligned to each other at directions away from $\langle 110\rangle$. The zoomed in optical images presented in Fig. 24 clearly describe the self-alignment nature of the hexagons near the precise $\langle 110\rangle$ directions. This method is completely based on visual inspection and does not require any measurement and therefore can be employed using only an optical microscope. The large misalignment at directions away from $\langle 110\rangle$ direction reduces the domain over which a closer inspection would be required to determine the precise $\langle 110\rangle$ direction. This technique is simple, robust and accurate. Additionally, it provides a longer length for aligning the edges of subsequent mask patterns. This method overcomes the shortcomings of the previous method where the mask edges needed to be aligned with respect to two corners. In this technique, the pre-fabricated reference line at the wafer end on the mask can be aligned with the notches of four circles along their diagonals. This not only reduces the efforts in alignment of subsequent mask, but also reduces the associated errors.

\section{Summary}

In this review article we have discussed all the techniques available till date to identify the crystallographic directions on $\operatorname{Si}\{100\}$ and $\operatorname{Si}\{110\}$ wafers. We tabulate all the techniques for an easy reference for the readers. 


\begin{tabular}{|c|c|c|c|c|}
\hline Authors & Wafer orientation & Structure & Alignment technique & Property of the method \\
\hline James et al. [76] & Si $\{100\}$ and $\operatorname{Si}\{110\}$ & Cleaved edge & $\begin{array}{l}\text { Cleave along the crystal- } \\
\text { lographic direction }\end{array}$ & $\begin{array}{l}\text { Space requirement This method requires scribed } \\
\text { lines and therefore full wafer cannot be used for } \\
\text { fabrication } \\
\text { Measurement required No } \\
\text { Accuracy Not accurate } \\
\text { Requires sophisticated equipment No } \\
\text { Remarks Accuracy depends on the skills of the han- } \\
\text { dler and the precision of the scribed line }\end{array}$ \\
\hline Ensell [104] & $\operatorname{Si}\{100\}$ & $\begin{array}{l}\text { Circular openings on } \\
\text { an arc }\end{array}$ & $\begin{array}{l}\text { After etching, choose } \\
\text { structures with mini- } \\
\text { mum distance between } \\
\text { the edges of adjacent } \\
\text { structures }\end{array}$ & $\begin{array}{l}\text { Space requirement Less } \\
\text { Measurement required Yes for better accuracy } \\
\text { Accuracy } 0.1^{\circ} \\
\text { Requires sophisticated equipment Yes if measurement } \\
\text { is needed } \\
\text { Remarks Simple technique }\end{array}$ \\
\hline $\begin{array}{l}\text { Vangbo and } \\
\text { Backlund [102] }\end{array}$ & Si $\{100\}$ and Si $\{110\}$ & Alignment fork & $\begin{array}{l}\text { After etching, inspect for } \\
\text { structures with symmet- } \\
\text { ric undercutting }\end{array}$ & $\begin{array}{l}\text { Space requirement Less } \\
\text { Measurement required No } \\
\text { Accuracy } 0.05^{\circ} \text { provided undercutting is constant } \\
\text { Requires sophisticated equipment No } \\
\text { Remarks Complex in design, requires visual inspec- } \\
\text { tion to find symmetric undercut structures, prone } \\
\text { to error due to varying undercut rate }\end{array}$ \\
\hline $\begin{array}{l}\text { Chang and } \\
\text { Huang [107] }\end{array}$ & $\operatorname{Si}\{100\}$ and $\operatorname{Si}\{110\}$ & Tapered ridges & $\begin{array}{l}\text { Look for merged structure } \\
\text { (target ridge) to deter- } \\
\text { mine the misalignment }\end{array}$ & $\begin{array}{l}\text { Space requirement More } \\
\text { Measurement required No } \\
\text { Accuracy } 0.05^{\circ}(100), 0.03^{\circ}(110) \\
\text { Requires sophisticated equipment No } \\
\text { Remarks Complex design pattern, tedious method }\end{array}$ \\
\hline Lai et al. [105] & $\operatorname{Si}\{100\}$ & $\begin{array}{l}\text { Rectangular and } \\
\text { squared openings on } \\
\text { an arc }\end{array}$ & $\begin{array}{l}\text { After etching choose } \\
\text { pattern with minimum } \\
\text { undercut }\end{array}$ & $\begin{array}{l}\text { Space requirement More } \\
\text { Measurement required Yes for better accuracy } \\
\text { Accuracy Prone to theta error, can be made more } \\
\text { accurate by patterning the same structures on } \\
\text { auxiliary sets as well } \\
\text { Requires sophisticated equipment Yes if measurement } \\
\text { is needed } \\
\text { Remarks Merging of structure occurs at directions } \\
\text { away from }\langle 110\rangle \text {. As a result the domain of inspec- } \\
\text { tion reduces to unmerged structures }\end{array}$ \\
\hline Chen et al. [109] & $\operatorname{Si}\{100\}$ & $\begin{array}{l}\text { Circular openings on } \\
\text { an arc }\end{array}$ & $\begin{array}{l}\text { After etching the corners } \\
\text { of the formed squares } \\
\text { merge due to undercut- } \\
\text { ting. The number of } \\
\text { such merged corners } \\
\text { decide the position of } \\
\langle 100\rangle \text { direction }\end{array}$ & $\begin{array}{l}\text { Space requirement Less } \\
\text { Measurement required None } \\
\text { Accuracy Good provided undercutting is constant } \\
\text { Requires sophisticated equipment No } \\
\text { Remarks Due to varying undercutting rate at different } \\
\text { structure, this method may give inaccurate results, } \\
\text { difficult to align subsequent mask }\end{array}$ \\
\hline Sajal et al. [110] & $\operatorname{Si}\{100\}$ & $\begin{array}{l}\text { Circular openings on } \\
\text { concentric arcs }\end{array}$ & $\begin{array}{l}\text { After etching, the notches } \\
\text { of the formed square } \\
\text { cavities aligns at precise } \\
\text { direction }\end{array}$ & $\begin{array}{l}\text { Space requirement More } \\
\text { Measurement required None } \\
\text { Accuracy Good } \\
\text { Requires sophisticated equipment No } \\
\text { Remarks Self aligning methodology makes the } \\
\text { crystallographic direction appear obvious, longer } \\
\text { length for aligning subsequent mask edges } \\
\text { reduces theta error }\end{array}$ \\
\hline Ciarlo [106] & $\operatorname{Si}\{110\}$ & $\begin{array}{l}\text { Rectangular openings } \\
\text { on an arc }\end{array}$ & $\begin{array}{l}\text { After etching choose the } \\
\text { rectangle with mini- } \\
\text { mum undercutting }\end{array}$ & $\begin{array}{l}\text { Space requirement More } \\
\text { Measurement required Yes for better accuracy } \\
\text { Accuracy Good } \\
\text { Requires sophisticated equipment Yes if measurement } \\
\text { is needed } \\
\text { Remarks Simple technique, but requires longer etch- } \\
\text { ing time }\end{array}$ \\
\hline James et al. [76] & $\operatorname{Si}\{110\}$ & $\begin{array}{l}\text { One circular opening } \\
\text { of diameter } 1 \mathrm{~mm}\end{array}$ & $\begin{array}{l}\text { Aligning of formed hexa- } \\
\text { gon with a dimension- } \\
\text { ally similar hexagon on } \\
\text { subsequent mask }\end{array}$ & $\begin{array}{l}\text { Space requirement Less } \\
\text { Measurement required None } \\
\text { Accuracy Good } \\
\text { Requires sophisticated equipment NO } \\
\text { Remarks Very simple, requires very less etching time, } \\
\text { uses less space, prone to theta error as aligning of } \\
\text { mask patterns is done only on one side of the wafer }\end{array}$ \\
\hline
\end{tabular}




\begin{tabular}{|c|c|c|c|c|}
\hline Authors & Wafer orientation & Structure & Alignment technique & Property of the method \\
\hline $\begin{array}{l}\text { Tseng and } \\
\text { Chang [103] }\end{array}$ & $\operatorname{Si}\{110\}$ & $\begin{array}{l}\text { Circular openings of } \\
\text { various diameters on } \\
\text { concentric arcs }\end{array}$ & $\begin{array}{l}\text { Alignment of notches of } \\
\text { adjacent hexagons }\end{array}$ & $\begin{array}{l}\text { Space requirement More } \\
\text { Measurement required None } \\
\text { Accuracy Good, but alignment with respect to cor- } \\
\text { ners makes it prone to error } \\
\text { Requires sophisticated equipment High magnification } \\
\text { microscope to visualise corner deviation of smaller } \\
\text { circles for better accuracy } \\
\text { Remarks Longer etching time, alignment of subse- } \\
\text { quent mask is done with respect to corners }\end{array}$ \\
\hline Sajal et al. [110] & $\operatorname{Si}\{110\}$ & $\begin{array}{l}\text { Circular openings on } \\
\text { concentric arcs }\end{array}$ & $\begin{array}{l}\text { After etching, the notches } \\
\text { of the formed hexagons } \\
\text { aligns precisely to each } \\
\text { other at the precise } \\
\langle 110\rangle \text { direction }\end{array}$ & $\begin{array}{l}\text { Space requirement More } \\
\text { Measurement required None } \\
\text { Accuracy Good } \\
\text { Requires sophisticated equipment No } \\
\text { Remarks Crystal direction becomes obvious as the } \\
\text { notches self-aligns itself at the precise direction, } \\
\text { longer length for subsequent alignment of prefab- } \\
\text { ricated line on subsequent mask }\end{array}$ \\
\hline
\end{tabular}

\section{Conclusions}

This review article is aimed at aggregating at one place, the different techniques proposed till date to precisely determine the crystallographic directions on $\mathrm{Si}\{100\}$ and $\mathrm{Si}\{110\}$ wafers. Different techniques including the cleaved-edge method and the use of pre-etched patterns to determine the crystal directions are discussed. Different geometric patterns are analysed based on their applicability on either $\mathrm{Si}\{100\}$ or $\mathrm{Si}\{110\}$ wafer. The basic technique to align the mask edges along the crystal directions is to use the wafer flat as the reference. However, given the uncertainty in the determination of wafer flat, it is not enough when high dimensional accuracy is desired. As a result alternate methodologies have been developed. One of them is by fabricating structures using the wafer flat as the reference and then using the edges of the structure with minimum undercut length as the accurate crystal direction. However, measurement of such undercut lengths which are very small in some cases is a tedious task as well as requires sophisticated equipment. To overcome these shortcomings, other techniques have been proposed which do not require the measurement of any kind. One of them is to by choosing structures whose corners merge after etching, another by choosing the structure with symmetric undercut etc. While these techniques are measurement free, still it comes with an associated errors due to varying undercut rate at different places on wafers, the difficulty in determining the target structures where undercutting is symmetric, etc. More recently, self-aligning techniques have been developed which makes the crystal direction appear obvious by virtue of the self-alignment nature of the structures. These techniques overcome the shortcomings of all the previous techniques in terms of being measurement free, robustness, simplicity in implementation and reduced efforts required for identifying the direction. These techniques can be employed without using any sophisticated equipment.

\section{Authors' contributions}

SSS and PP carried out literature search and wrote the manuscript. AKP, YX and $\mathrm{KS}$ reviewed/edited the manuscript. All authors read and approved the final manuscript.

\section{Authors' information}

Sajal Sagar Singh completed his Bachelor's degree in the Department of Mechanical and Aerospace Engineering at Indian Institute of Technology (IIT) Hyderabad, India in 2015. Currently, he is a final year Master's student at IIT Hyderabad. His research interest lies in the field of linear and nonlinear dynamics, sensors and actuators, microfabrication techniques etc. He has published more than ten research papers in peer-reviewed international journals of high impact factor.

Prem Pal did his M.Tech in Solid State Material (SSM) in December 1999 and Ph.D. in the area of MEMS in December 2004, both from Indian Institute of Technology Delhi (IIT Delhi), India. The Foundation for Innovation and Technology Transfer (FITT), IIT Delhi awarded his thesis for "Best Industry Relevant Ph.D. Project-2005". From July 2005 to June 2006, he worked as Postdoctoral Researcher at Yonsei Microsystems Laboratory (YML), School of Mechanical Engineering, Yonsei University Seoul, South Korea. He worked in Sato laboratory, Department of Micro-Nano Systems Engineering, Nagoya University, Nagoya, Japan: from July 2006 to March 2008 as COE Scientist and from March 2008 to March 2010 as JSPS Fellow. He joined the Department of Physics, Indian Institute of Technology Hyderabad in April 2010 as Assistant Professor Presently, he is working as Associate Professor. He has published more than 50 papers in peer reviewed international journals of high impact factors. His research interests include MEMS technology, MEMS based sensors, Silicon Micromachining, Thin films for MEMS.

Ashok Kumar Pandey received the B.E. degree in mechanical engineering from Bhilai Institute of Technology, Durg, India, in 2001, and the M.S. and Ph.D. degrees in mechanical engineering from Indian Institute of Science, Bengaluru, India, in 2003 and 2007 respectively. He joined the Department of Mechanical and Aerospace Engineering, Indian Institute of Technology Hyderabad in July 2010. Currently, he is an Associate Professor in the same department. His research interest includes nonlinear dynamics, vehicle dynamics and micro and nanomechanics.

Yan Xing received the B.S., M.S., and Ph.D. degrees in mechanical engineering from Southeast University, Nanjing, China, in 1993, 1996, and 2001, respectively. He was with the Department of Micro-Nano Systems Engineering, Nagoya University, Nagoya, Japan from 2005 to 2007. He is currently a full Professor with the Department of Mechanical Engineering, Southeast University, Nanjing, China. He has been studying numerical simulation technologies with respect to MEMS CAD, semiconductor processes, and integrated information systems in automation.

Kazuo Sato graduated from Yokohama National University, Japan and received his BS Degree in 1970. He worked with Hitachi Co. Ltd. in a period of 1970-1994. During this period, he received his PhD degree from the University of Tokyo in 1982. He started Si-micromachining research in 1983 and published 128 international journal papers in MEMS technologies. He 
joined Nagoya University in 1994 as a full professor at Department of Micro Systems Engineering. He became Emeritus professor of Nagoya University in 2012, and currently is a professor at Aichi Institute of Technology, Japan. His research interests include bulk micromachining of Si and quartz, mechanical characterization of MEMS materials, and MEMS applications. He co-chaired IEEE MEMS-97 conference. He was the editor in Asia (2008-2012) and currently is an editorial board member of the Journal of Micromechanics and Microengineering. He is the founding chair of the Division of Micro/Nano Science and Engineering in the Japan Society of Mechanical Engineers (JSME), and Honorary Member of the JSME. He also is a senior member of IEEJ, and a fellow of JSPE, and a member of IEEE.

\section{Author details}

${ }^{1}$ Department of Mechanical and Aerospace Engineering, Indian Institute of Technology Hyderabad, Kandi Sangareddy, India. ${ }^{2}$ MEMS and Micro/Nano Systems Laboratory, Department of Physics, Indian Institute of Technology Hyderabad, Kandi Sangareddy, India. ${ }^{3}$ School of Mechanical Engineering, Southeast University Nanjing, Nanjing, China. ${ }^{4}$ Department of Mechanical Engineering, Aichi Institute of Technology, Toyota, Aichi, Japan.

\section{Acknowledgements}

This work was supported by research grant from the Council of Scientific and Industrial Research (CSIR, Ref: 03(1320)/14/EMR-II), New Delhi, India and a Research Project BY2015070-06 Jiaungsu Povince, China. One of the authors 'Dr. Prem Pal' gratefully acknowledges financial support from Japan International Cooperation Agency (JICA) for visiting Sato lab (AIT Toyota) in Oct 2015 in regard to collaborative research work. Sincere thanks to Ms. Michiko Shindo (Secretory to Prof. K. Sato) for her assistance in obtaining permissions to reproduce some figures from published papers.

\section{Competing interests}

The authors declare that they have no competing interests.

Received: 22 March 2016 Accepted: 4 May 2016 Published online: 11 June 2016

\section{References}

1. Ashok A, Pal P (2015) Silicon micromachining in 25 wt\% TMAH without and with surfactant concentrations ranging from $\mathrm{ppb}$ to $\mathrm{ppm}$. Microsyst Technol 1-8. doi:10.1007/s00542-015-2699-9

2. Lee S, Park S, Cho D (1999) The surface/bulk micromachining (SBM) process: a new method for fabricating released microelectromechanical systems in single crystal silicon. J Microelectromech Syst 8:409-416

3. Frühauf J (2005) Shape and functional elements of the bulk silicon microtechnique: a manual of wet-etched silicon structures. Springer, Berlin

4. Pal P, Chandra S (2004) Bulk-micromachined structures inside anisotropically etched cavities. Smart Mater Struct 13:1424-1429

5. Tellier CR, Charbonnieras AR (2003) Characterization of the anisotropic chemical attack of (hhl) silicon plates in a TMAH 25 wt\% solution: micromachining and adequacy of the dissolution slowness surface. Sens Actuators A Phys 105:62-75

6. Schnakenberg U, Benecke W, Lochel B (1990) $\mathrm{NH}_{4} \mathrm{OH}$-based etchant for silicon micromachining. Sens Actuators A Phys 23:1031-1035

7. Zubel I, Kramkowska M (2009) Possibilities of extension of 3D shapes by bulk micromachining of different Si (hkl) substrates. J Micromech Microeng 15:485-493

8. Pal P, Sato K (2015) A comprehensive review on convex and concave corners in silicon bulk micromachining based on anisotropic wet chemical etching. Micro Nano Syst Lett 3:1-42

9. Gad-el-Hak M (ed) (2002) The MEMS handbook. CRC Press LLC, Boca Raton

10. Elwenspoek M, Jansen H (1998) Silicon micromachining. Cambridge University Press, UK

11. Takahata K (2013) Advances in micro/nano electromechanical systems and fabrication technologies. InTech, Rijeka
12. Lindroos V, Tilli M, Lehto A, Motooka T (2010) Handbook of silicon based MEMS materials and technologies. William Andrew Publishing, Norwich

13. Hsu TR (2003) MEMS \& microsystems: design and manufacture. Tata McGraw-Hill Publishing Company Ltd, New Delhi

14. Madou MJ (2002) Fundamentals of microfabrication: the science of miniaturization, 2nd edn. CRC Press, Boca Raton

15. Varadan VK, Vinoy KJ, Gopalakrishnan S (2006) Smart material systems and MEMS: design and development methodologies. Wiley, New York

16. Lang W (1996) Silicon microstructuring technology. Mater Sci Eng R Rep 17:1-55

17. Bustillo JM, Howe RT, Muller RS (1998) Surface micromachining for microelectromechanical systems. IEEE Proc 86:1552-1574

18. Bhatt V, Pal P, Chandra S (2005) Feasibility study of RF sputtered ZnO film for surface micromachining. Surf Coat Technol 198:304-308

19. Kovacs GT, Maluf NI, Petersen KE (1998) Bulk micromachining of silicon. IEEE Proc 86:1536-1551

20. Petersen KE (1982) Silicon as a mechanical material. IEEE Proc 70:420-457

21. Jansen H, Gardeniers H, Boer MD, Elwenspoek M, Fluitman J (1996) A survey on the reactive ion etching of silicon in microtechnology. $J$ Micromech Microeng 6:14-28

22. Oehrlein GS (1990) Reactive ion etching. In: Rossnagel SM, Westwood WD, Haber JJ (eds) Handbook of plasma processing technologyfundamentals, etching, deposition, and surface interactions. Noyes, Park Ridge

23. Coburn JW, Winters HF (1979) Plasma etching-a discussion of mechanisms. J Vaccum Sci Technol 16:391-403

24. Larmer F, Schilp P (1994) Method of anisotropically etching silicon. German Patent DE 4(241):045

25. Jiang E, Keating A, Martyniuk M, Prasad K, Faraone L, Jiang JM (2012) Characterization of low-temperature bulk micromachining of silicon using an $\mathrm{SF}_{6} / \mathrm{O}_{2}$ inductively coupled plasma. J Micromech Microeng 22:095005

26. Hynes AM, Ashraf H, Bhardwaj JK, Hopkins J, Johnston I, Shepherd JN (1999) Recent advances in silicon etching for MEMS using the ASE process. Sens Actuators A Phys 74:13-17

27. Teng J, Prewett PD (2005) Focused ion beam fabrication of thermally actuated bimorph cantilevers. Sens Actuators A Phys 123-124:608-613

28. Walker CK, Narayanan G, Knoepfle H, Capara J, Glenn J, Hungerford A, Bloomstein TM, Palmacci ST, Stern MB, Curtin JE (1997) Laser micromachining of silicon: a new technique for fabricating high quality terahertz waveguide components. In: Proceedings of 8 th international symposium on space terahertz technology, Harvard University, p 358

29. Schwartz B, Robbins H (1976) Chemical etching of silicon. J Electrochem Soc 123(12):1903-1909

30. Zandi K, Arzi E, Izadi N, Mohajerzadeh S, Haji S, Abdi Y, AsI Soleimani E (2006) Study of bulk micromachining for $\langle 100\rangle$ silicon. Eur Phys J Appl Phys 35:7-12

31. Lee DB (1969) Anisotropic etching of silicon. J Appl Phys 40:4569-4575

32. Bean KE (1978) Anisotropic etching of silicon. IEEE Trans Electron Devices 25:1185-1193

33. Seidel H, Csepregi L, Heuberger A, Baumgartel H (1990) Anisotropic etching of crystalline silicon in alkaline solutions I: orientation dependence and behavior of passivation layers. J Electrochem Soc 137(11):3612-3626

34. Sato K, Shikida M, Matsushima Y, Yamashiro T, Asaumi K, Iriye Y, Yamamoto M (1998) Characterization of orientation-dependent etching properties of single-crystal silicon: effects of $\mathrm{KOH}$ concentration. Sens Actuators A Phys 61:87-93

35. Dutta S, Imran Md, Kumar P, Pal R, Datta P, Chatterjee R (2011) Comparison of etch characteristics of $\mathrm{KOH}, \mathrm{TMAH}$ and EDP for bulk micromachining of silicon (110). Microsyst Technol 17:1621-1628

36. Powell O, Harrison HB (2001) Anisotropic etching of 100 and 110 planes in (100) silicon. J Micromech Microeng 11:217-220

37. Tanaka H, Yamashita S, Abe Y, Shikida M, Sato K (2004) Fast etching of silicon with a smooth surface in high temperature ranges near the boiling point of KOH solution. Sens Actuators A Phys 114:516-520

38. Matsuoka M, Yoshida Y, Moronuki M (1992) Preparation of silicon thin diaphragms free from micropyramids using anisotropic etching in $\mathrm{KOH}$ solution. J Chem Eng 25:735-740 
39. Baryeka I, Zubel I (1995) Silicon anisotropic etching in $\mathrm{KOH}$-isopropanol etchant. Sens Actuators A Phys 48:229-238

40. Shikida M, Sato K, Tokoro K, Uchikawa D (2000) Differences in anisotropic etching properties of $\mathrm{KOH}$ and TMAH solutions. Sens Actuators A Phys 80:179-188

41. Backlund $Y$, Rosengren L (1992) New shapes in (100) Si using KOH and EDP etches. J Micromech Microeng 27:5-9

42. Pal P, Ashok A, Haldar S, Xing Y, Sato K (2015) Anisotropic etching in low concentration $\mathrm{KOH}$ : effects of surfactant concentration. Micro Nano Lett 10(4):224-228

43. Tanaka H, Cheng D, Shikida M, Sato K (2006) Characterization of anisotropic wet etching properties of single crystal silicon: effects of ppblevel of $\mathrm{Cu}$ and $\mathrm{Pb}$ in $\mathrm{KOH}$ solution. Sens Actuators A Phys 128:125-131

44. Tabata O, Asahi R, Funabashi H, Shimaoka K, Sugiyama S (1992) Anisotropic etching of silicon in TMAH solutions. Sens Actuators A Phys 34(1):51-57

45. Tang B, Shikida M, Sato K, Pal P, Amakawa H, Hida H, Fukuzawa K (2010) Study of surfactant-added TMAH for the applications in DRIE + wet etching based micromachining. J Micromech Microeng 20:065008

46. Mukhiya R, Bagolini A, Margesin B, Zen M, Kal S (2006) <100 > bar corner compensation for CMOS compatible anisotropic TMAH etching. J Micromech Microeng 16:2458-2462

47. Sato K, Shikida M, Yamashiro T, Asaumi K, Iriye Y, Yamamoto M (1999) Anisotropic etching rates of single-crystal silicon for TMAH water solution as a function of crystallographic orientation. Sens Actuators A Phys 73:131-137

48. Pal P, Sato K, Gosalvez MA, Tang B, Hida H, Shikida M (2010) Fabrication of novel microstructures based on orientation dependent adsorption of surfactant molecules in TMAH solution. J Micromech Microeng 21(1):015008

49. Pal P, Sato K (2010) Fabrication methods based on wet etching process for the realization of silicon MEMS structures with new shapes. Microsyst Technol 16(7):1165-1174

50. Gosalvez MA, Tang B, Pal P, Sato K, Kimura Y, Ishibashi K (2009) Orientation and concentration dependent surfactant adsorption on silicon in aqueous alkaline solutions: explaining the changes in the etch rate roughness and undercutting for MEMS applications. J Micromech Microeng 19(12):125011

51. Steinsland E, Finstad T, Hanneborg A (2000) Etch rates of (100), (111), and (110) single-crystal silicon in TMAH measured in situ by laser reflectance interferometry. Sens Actuators A Phys 86:73-80

52. Tang B, Yao MQ, Tan G, Pal P, Sato K, Su W (2014) Smoothness control of wet etched Si\{100\} surfaces in TMAH + Triton. Key Eng Mater 609:536-541

53. Shikida M, Masuda T, Uchikawa D, Sato K (2001) Surface roughness of single-crystal silicon etched by TMAH solution. Sens Actuators A Phys 90(3):223-231

54. Tang B, Sato K, Zhang D, Cheng Y (2014) Fast Si (100) etching with a smooth surface near the boiling temperature in surfactant modified tetramethylammonium hydroxide solutions. Micro Nano Lett 9(9):582-584

55. Gosalvez MA, Pal P, Ferrando N, Hida H, Sato K (2011) Experimental procurement of the complete $3 \mathrm{D}$ etch rate distribution of $\mathrm{Si}$ in anisotropic etchants based on vertically micromachined wagon wheel samples. J Micromech Microeng 21:125007

56. Tang B, Pal P, Gosalvez MA, Shikida M, Sato K, Amakawa H, Itoh S (2009) Ellipsometry study of the adsorbed surfactant thickness on Si\{110\} and $\mathrm{Si}\{100\}$ and the effect of pre-adsorbed surfactant layer on etching characteristics in TMAH. Sens Actuators A Phys 156:334-341

57. Chung GS (2005) Anisotropic etching characteristics of Si in tetramethylammonium hydroxide: isopropyl alcohol: pyrazine solutions. J Korean Phys Soc 46(5):1152-1156

58. Choi WK, Thong JTL, Luo P, Tan CM, Chua TH, Bai Y (1998) Characterisation of pyramid formation arising from the TMAH etching of silicon. Sens Actuators A Phys 71:238-243

59. Resnik D, Vrtacnik D, Aljancic U, Amon S (2003) Effective roughness reduction of 100 and 311 planes in anisotropic etching of 100 silicon in 5 \% TMAH. J Micromech Microeng 13:26-34

60. Sakaino K, Adachi S (2001) Study of Si(100) surfaces etched in TMAH solution. Sens Actuators A Phys 88:71-78
61. Pal P, Sato K, Gosalvez MA (2012) Etched profile control in anisotropic etching of silicon by TMAH + Triton. J Micromech Microeng 22(6):065013

62. Resnik D, Vrtacnik D, Aljancic U, Amon S (2000) Wet etching of silicon structures bounded by (311) sidewalls. Microelectron Eng 51:555-566

63. Holke A, Henderson HT (1999) Ultra-deep anisotropic etching of (110) silicon. J Micromech Microeng 9:51-57

64. Thong JTL, Choi WK, Chong CW (1997) TMAH etching of silicon and the interaction of etching parameters. Sens Actuators A Phys 63(3):243-249

65. Seidel H, Csepregi L, Heuberger A, Baumgartel H (1990) Anisotropic etching of crystalline silicon in alkaline solutions II: influence of dopants. J Electrochem Soc 137:3626-3632

66. Wu MP, Wu QH, Ko WH (1986) A study on deep etching of silicon using ethylenediamine-pyrocatechol-water. Sens Actuators A Phys 9:333-343

67. Reisman A, Berkenblit M, Chan SA, Kaufmann FB, Green DC (1979) The controlled etching of silicon in catalyzed ethylene-diamine-pyrochatechol-water solutions. J Electrochem Soc Solid-State Sci Technol 126:1406-1415

68. Kern W (1978) Chemical etching of silicon, germanium, gallium arsenide, and gallium phosphide. RCA Rev 39:278-307

69. Declercq MJ, Gerzberg L, Meindl JD (1975) Optimization of the hidrazine-water solution for anisotropic etching of silicon in integrated circuit technology. J Electrochem Soc Solid State Sci 122:545-552

70. Schnakenberg U, Benecke W, Lochel B (1990) $\mathrm{NH}_{4} \mathrm{OH}$-based etchant for silicon micromachining. Sens Actuators A Phys 23:1031-1035

71. Clarck LD, Lund JL, Edell DJ (1988) Cesium hydroxide (CsOH): a useful etchant for micromachining silicon. In: Tech. digest, IEEE solid state sensor and actuator workshop (Hilton Head Island, SC), pp 5-8

72. Pal P, Singh SS (2013) A simple and robust model to explain convex corner undercutting in wet bulk micromachining. Micro Nano Syst Lett 1(1):1-6

73. Pal P, Singh SS (2013) A new model for the etching characteristics of corners formed by Si\{111\} planes on Si\{110\} wafer surface. Engineering 5(11):1-8

74. Kandlikar SG, Grande WJ (2003) Evolution of microchannel flow passages-thermohydrolic performance and fabrication technology. Heat Transf Eng 24:3-17

75. Koo JM, Jian L, Zhang L, Kenny T, Santiago J, Goodson K (2001) Modeling of two-phase microchannel heatsinks for VLSI chips In: 14th IEEE international conference on micro electro mechanical systems, MEMS, IEEE, Interlaken, pp 422-426

76. James TD, Parish G, Winchester KJ, Musca CA (2006) A crystallographic alignment method in silicon for deep, long microchannel fabrication. J Micromech Microeng 16(10):2177

77. Pal P, Sato K (2009) Various shapes of silicon freestanding microfluidic channels and microstructures in one step lithography. J Micromech Microeng 19(5):055003

78. Abedinov N, Grabiec P, GotszalkT, Tz Ivanov, Voigt J, Rangelow IW (2001) Micromachined piezoresistive cantilever array with integrated resistive microheater for calorimetry and mass detection. JVaccum Sci Technol A 19:2884-2888

79. Saya D, Belaubre P, Mathieu F, Lagrange D, Pourciel JB, Bergaud C (2005) Si-piezoresistive microcantilevers for highly integrated parallel force detection applications. Sensors Actuators A Phys 123:23-29

80. Lee JH, Hwang KS, Park J, Yoon KH, Yoon DS, Kim TS (2005) Immunoassay of prostate-specific antigen (PSA) using resonant frequency shift of piezoelectric nanomechanical microcantilever. Biosens Bioelectron 20:2157-2162

81. Battiston FM, Ramseyer JP, Lang HP, Baller MK, Gerber C, Gimzewski JK, Meyer E, Guntherodt HJ (2001) A chemical sensor based on a microfabricated cantilever array with simultaneous resonance frequency and bending readout. Sens Actuators B Chem 77:122-131

82. Wee KW, Kang GY, Park J, Kang JY, Yoon DS, Parkb JH, Kim TS (2005) Novel electrical detection of label-free disease marker proteins using piezoresistive self-sensing micro-cantilevers. Biosens Bioelectron 20:1932-1938

83. Pruitt BL, Kenny TW (2003) Piezoresistive cantileveres and measurement systems for characterizing low force electrical contacts. Sens Actuators A Phys 104:68-77 
84. Baller MK, Lang HP, Fritz J, Gerber C, Gimzewski JK, Drechsler U, Rothuizen H, Despont M, Vettiger P, Battiston FM, Ramseyer JP (2000) A cantilever array-based artificial nose. Ultramicroscopy 82(1):1-9

85. Pal P, Sato K (2009) Suspended Si microstructures over controlled depth micromachined cavities for MEMS based sensing devices. Sens Lett 7:11-16

86. Zhang Y, Tadigadapa S (2004) Calorimetric biosensors with integrated microfluidic channels. Biosens Bioelectron 19:1733-1743

87. Winter W, Hohne GWH (2003) Chip-calorimeter for small samples. Thermochim Acta 403:43-53

88. Van Herwaarden AW, Van Duyn DC, Van Oudheusden BW, Sarro PM (1989) Integrated thermopile sensors. Sens Actuators A Phys 22:621-630

89. Sarro PM, van Hexwaarden AW, van der Vlist W (1994) A silicon-silicon nitride membrane fabrication process for smart thermal sensors. Sens Actuators A Phys 42(1):666-671

90. Wang CC, Gogoi BP, Monk DJ, Mastrangelo CH (2000) Contaminationinsensitive differential capacitive pressure sensors. IEEE J Microelectromech Syst 9:538-543

91. Tiren J, Tenerz L, Hok B (1989) A batch-fabricated non-reverse valve with cantilever beam manufactured by micromachining of silicon. Sens Actuators 18:389-396

92. Oh KW, Ahn CH (2006) A review of microvalves. J Micromech Microeng 16(5):R13-R39

93. Bien DCS, Mitchell SJN, Gamble HS (2003) Fabrication and characterization of a micromachined passive valve. J Micromech Microeng 13:557-562

94. Au AK, Lai H, Utela BR, Folch A (2011) Microvalves and Micropumps for BioMEMS. Micromachines 2:179-220

95. Tsai NC, Sue CY (2007) Review of MEMS-based drug delivery and dosing systems. Sens Actuators A Phys 134:555-564

96. Nisar A, Afzulpurkar N, Mahaisavariya B, Tuantranont A (2008) MEMSbased micropumps in drug delivery and biomedical applications. Sens Actuators B Chem 130:917-942

97. Laser DJ, Santiago JG (2004) A review of micropumps. J Micromech Microeng 14:35-64

98. Woias P (2005) Micropumps-past, progress and future prospects. Sens Actuators B Chem 105:28-38

99. Sharma J, Krishanapura N, Das GA (2012) Fabrication of low pull-in voltage RF MEMS switches on glass substrate in recessed CPW configuration for V-band application. J Micromech Microeng 22:025001

100. Pal P, Sato K, Chandra S (2007) Fabrication techniques of convex corners in (100)-silicon wafer using bulk micromachining: a review. J Micromech Microeng 17:R11-R13

101. Gravesen P, Branebjerg J, Jensen OS (1993) Microfluidics-a review. J Mioromech Microeng 3:168-182

102. Vangbo M, Backlund Y (1996) Precise mask alignment to the crystallographic orientation of silicon wafers using wet anisotropic etching. J Micromech Microeng 6(2):279

103. Tseng FG, Chang KC (2002) Precise [100] crystal orientation determination on $\langle 110\rangle$-oriented silicon wafers. J Micromech Microeng 13(1):47

104. Ensell G (1996) Alignment of mask patterns to crystal orientation. Sens Actuators A Phys 3(1):345-348

105. Lai JM, Chieng WH, Huang YC (1998) Precision alignment of mask etching with respect to crystal orientation. J Micromech Microeng 8(4):327

106. Ciarlo DR (1992) A latching accelerometer fabricated by the anisotropic etching of (110) oriented silicon wafers. J Micromech Microeng 2(1):10

107. Chang WH, Huang YC (2005) A new pre-etching pattern to determine $\langle 110\rangle$ crystallographic orientation on both (100) and (110) silicon wafers. Microsyst Technol 11(2-3):117-128

108. Chen PH, Hsieh CM, Peng HY, Chyu MK (2000) Precise mask alignment design to crystal orientation of (100) silicon wafer using wet anisotropic etching. In: Micromachining and microfabrication international society for optics and photonics, pp 462-466
109. Singh SS, Veerla S, Sharma V, Pandey AK, Pal P (2016) Precise identification of $\langle 100\rangle$ directions on Si 001 wafer using a novel self-aligning pre-etched technique. J Micromech Microeng 26(2):025012

110. Singh SS, Avvuru NV, Veerla S, Pandey AK, Pal P (2016) A measurement free pre-etched pattern to identify the $\langle 110\rangle$ directions on Si\{110\} wafer. Microsys Technol pp 1-7. doi:10.1007/s00542-016-2984-2

111. Pal P, Gosalvez MA, Sato K (2010) Silicon micromachining based on surfactant-added tetramethyl ammonium hydroxide: etching mechanism and advanced application. Japan J Appl Phys 49:056702

112. Gosalvez MA, Pal P, Tang B, Sato K (2010) Atomistic mechanism for the macroscopic effects induced by small additions of surfactants to alkaline etching solutions. Sens Actuator A Phys 157(1):91-95

113. Sekimura M (1999) Anisotropic etching of surfactant-added TMAH solution. In: Proceedings 12th IEEE micro-electro-mechanical systems conference, Orlando, pp 650-655

114. Pal P, Sato K, Gosalvez MA, Shikida M (2007) Study of rounded concave and sharp edge convex corners undercutting in CMOS compatible anisotropic etchants. J Micromech Microeng 17:2299-2307

115. Resnik D, Vrtacnik D, Aljancic U, Mozek M, Amon S (2005) The role of Triton surfactant in anisotropic etching of 110 reflective planes on (100) silicon. J Micromech Microeng 15:1174-1183

116. Yang CR, Yang CH, Chen PY (2005) Study on anisotropic silicon etching characteristics in various surfactant-added tetramethylammonium hydroxide water solutions. J Micromech Microeng 15:202837

117. Sarro PM, Brida D, Vlist W, Brida S (2000) Effect of surfactant on surface quality of silicon microstructures etched in saturated TMAHW solutions. Sens Actuator A Phys 85:340-345

118. Pal P, Sato K, Gosalvez MA, Kimura Y, Ishibashi K, Niwano M, Hida H, Tang B, Itoh S (2009) Surfactant adsorption on single crystal silicon surfaces in TMAH solution: orientation-dependent adsorption detected by in situ infra-red spectroscopy. J Microelectromech Syst 18:1345-1356

119. Pal P, Sato K, Shikida M, Gosalvez MA (2009) Study of corner compensating structures and fabrication of various shapes of MEMS structures in pure and surfactant added TMAH. Sens Actuators A Phys 154:192203

120. XuYW, Michael A, KwokCY (2011) Formation of ultra-smooth $45^{\circ}$ micromirror on (100) silicon with low concentration TMAH and surfactant: techniques for enlarging the truly $45^{\circ}$ portion. Sens Actuators A Phys 166:16471

121. Rola KP, Ptasiski K, Zakrzewski A, Zubel I (2014) Silicon 45 micromirrors fabricated by etching in alkaline solutions with organic additives. Microsys Technol 20:221-226

122. Yagyu H, Yamaji T, Nishimura M, Sato K (2010) Forty-five degree micromirror fabrication using silicon anisotropic etching with surfactantadded tetramethylammonium hydroxide solution. Japan J Appl Phys 49:096503

123. Pal P, Haldar S, Singh SS, Ashok A, Xing Y, Sato K (2014) A detailed investigation and explanation to the appearance of different undercut profiles in $\mathrm{KOH}$ and TMAH. J Micromech Microeng 24:095026

124. Gosalvez MA, Li Y, Ferrando N, Pal P, Sato K, Xing Y (2016) Fluctuations during anisotropic etching: local recalibration and application to Si\{110\}. J Microelectromech Syst 25:1-11

\section{Submit your manuscript to a SpringerOpen ${ }^{\circ}$ journal and benefit from:}

- Convenient online submission

- Rigorous peer review

- Immediate publication on acceptance

- Open access: articles freely available online

- High visibility within the field

- Retaining the copyright to your article

Submit your next manuscript at springeropen.com 